\title{
Three-Dimensional Dynamic Analyses of Track-Embankment-Ground System Subjected to High Speed Train Loads
}

\author{
Qiang Fu and Changjie Zheng \\ Key Laboratory of Ministry of Education for Geomechanics and Embankment Engineering, Hohai University, Nanjing 210098, China \\ Correspondence should be addressed to Qiang Fu; fuqutdhhu@163.com
}

Received 28 November 2013; Accepted 19 January 2014; Published 26 February 2014

Academic Editors: Z. Guan, S. Kaewunruen, and E. K. Zavadskas

Copyright (C) 2014 Q. Fu and C. Zheng. This is an open access article distributed under the Creative Commons Attribution License, which permits unrestricted use, distribution, and reproduction in any medium, provided the original work is properly cited.

\begin{abstract}
A three-dimensional finite element model was developed to investigate dynamic response of track-embankment-ground system subjected to moving loads caused by high speed trains. The track-embankment-ground systems such as the sleepers, the ballast, the embankment, and the ground are represented by 8-noded solid elements. The infinite elements are used to represent the infinite boundary condition to absorb vibration waves induced by the passing of train load at the boundary. The loads were applied on the rails directly to simulate the real moving loads of trains. The effects of train speed on dynamic response of the system are considered. The effect of material parameters, especially the modulus changes of ballast and embankment, is taken into account to demonstrate the effectiveness of strengthening the ballast, embankment, and ground for mitigating system vibration in detail. The numerical results show that the model is reliable for predicting the amplitude of vibrations produced in the track-embankment-ground system by high-speed trains. Stiffening of fill under the embankment can reduce the vibration level, on the other hand, it can be realized by installing a concrete slab under the embankment. The influence of axle load on the vibration of the system is obviously lower than that of train speed.
\end{abstract}

\section{Introduction}

In recent years, with the rapid development of high speed railway in China, the issue on dynamic response of soil and environment vibration induced by the moving trains has been paid more attention, and the researchers have paid attention to the dynamic effects associated with train-track interaction [1-8]. A significant trendy in this context is the high speed and self-weight of railway trains, which impose heavier loads on the tracks [9]. The moving high speed train often produces significant ground vibrations, especially at the resonance condition. Thus, how to avoid resonance and reduce the vibrations has become a key researching issue.

The soil vibration problem was first studied by Lamb in 1904, whose pioneering research mainly focused on the dynamic response of elastic half-space and elastic body with infinite boundary generated by stationary loads or approximately stationary loads [9]. The moving-load effects have been studied for beam structures and beams on Winkler foundation [10, 11]. A prediction model developed by
Krylov [12] was used [13, 14]. The steady-state vibration of a periodically supported beam on an elastic half-space under a uniformly moving harmonic load has been studied in [15]. Krylov's model is valid when the train speed near the critical phase velocity of the coupled track-soil system, as the quasi-static excitation, is dominant in this case [16-18]. Sheng et al. [19] studied the ground vibration generated by a harmonic load that moves along a railway track. Kaynia et al. [2], along with their presentation of measurements, presented the development of an FEM model composed of a moving load on a railway/embankment structure, which was coupled to a layered ground model at a series of points via the Green's functions. Kargarnovin et al. [20] have studied the response of infinite beams supported by nonlinear viscoelastic foundations subjected to harmonic moving loads. In $[21,22]$, a closed-form displacement response of beams on viscoelastic foundations has been presented for two cases of a line moving load and a concentrated moving load. Takemiya and Bian [23] and Picoux and Le Houédec [24] studied 
the dynamic responses of a track system on the ground subjected to a moving train load. In $[25,26]$, an analytical approach was used to investigate dynamic responses of a track system and the poroelastic half-space soil medium subjected to a moving point load under three-dimensional condition. In [27], Cao et al. investigated the vibrations of railway tracks on a poroelastic half-space generated by moving trains through a vehicle-track-ground coupling model. In [28], the dynamic response of a fully saturated poroelastic half-space due to accelerating or decelerating trains is investigated by a semianalytical method. In [29], the proposed approach was verified by the semianalytical solutions for a $3 \mathrm{D}$ saturated half space subjected to a moving load. Sheng et al. [30] also use a FEM/BEM approach for the analysis of ground vibration produced by trains. Galvín and Domínguez [31,32] presented a general and fully three-dimensional model for analysis of the soil motion and the effects of HST (high speed train) passage on nearby surface and underground structures. In [33], a general and fully three-dimensional multibody finite element boundary element model, formulated in the time domain to predict vibrations due to train passage at the vehicle, the track, and the free field, is presented. Kaynia et al. [2] and Takemiya and Bian [23] have done some works specifically to railway embankments by using the boundary element method. Hall [34] and Ju et al. [35] used the finite element method to perform three-dimensional time domain simulation for train-induced vibrations of embankment on layered ground. The rails, sleepers, ballast, embankment, and layered ground were all described in detail. The elastically distributed wheel load has been considered by Krylov et al. [36, 37] and Takemiya [38]. However, they have not proceeded to investigate the vehicles traveling effect under various speeds. By using Green's functions for a layered halfspace, or combined with a BEM FEM method, different authors have proposed approaches for analyzing soil induced vibrations due to moving loads. Most of the existing papers discussed the track-soil interaction problem in the frequency wave number domain by using Fourier transformation. The moving point loads were applied on the nodes in the beam elements for simulating the rail. However, the dynamic response of rack-embankment-ground system cannot be obtained in time domain with simulation accurately. For three-dimensional finite element models, the railway track is represented by 8-noded solid elements, the dynamic response can be obtained in the simulation. The validation of the simulation model is important. Kaewunruen and Remennikov [39] have dealt with the application of vibration measurements and finite element model updating to the assessment of ballasted rail track sleepers and indicated that the model proved its effectiveness for predicting the free vibration characteristics of in situ sleepers under different circumstances. In [40], the finite element model of the railway concrete sleeper was previously established and validated against experimental data by the authors.

In this research, the dynamic three-dimensional (3D) finite element program ABAQUS was chosen for creating the models used to simulate the vibration of track-embankmentground system induced by high speed train moving on the track system. The elements on the rail top are referred to as loading unit, and the moving loads were applied on the loading unit directly to simulate the train moving load. Four train speeds $(60,80,100$, and $120 \mathrm{~m} / \mathrm{s}$, resp.) were considered. These speeds were smaller, closer, or greater than the Rayleigh wave speed of the ground. The three-dimensional model analyses are presented in both the time and frequency domains. The effect of material parameters, especially the modulus changes of ballast, embankment, fill, and soil on the dynamic response of track system was taken into account. The ground vibration characteristics of track-ballast-embankment-ground system are analyzed under different train moving speeds.

\section{Numerical Model of Track-Embankment- Ground System}

2.1. Train Load Model. The train axle load and geometry were shown in Figure 1. For the three-dimensional models, the elements in the rail are referred to as loading unit, and the moving unit loads were applied on the 8-noded solid elements simulating the rail. In this case, the load distribution $P$ from the rail was calculated from the static solution of train axle load $F$ acting on the element $\Delta S$, the $P$ is given by

$$
P=\frac{F}{\Delta S},
$$

where, $F$ is $70 \mathrm{KN}$ and $\Delta S$ is $0.02 \mathrm{~m}^{2}$, so the calculated distribution load $P$ is $3.75 \mathrm{MPa}$. The load $P$ was then applied directly on the rail elements with time shifts corresponding to the train speed. All these loading models generate stress waves just from the main source, that is, the track structure response. Other sources such as rail defects, unsteady riding of the vehicle, and variable support were not considered. All the finite element analyses in this project were performed in the time domain using direct time integration. The time step of the analyses was set to be automatic and corresponded with vibration characteristic of the whole system.

2.2. Finite Element Model. The model was set to $130 \mathrm{~m}$ in length. This was assumed as an initial estimate since only the response close to the track was considered. The threedimensional model, consisted of rails, sleepers, ballast, and embankment, measured $45 \times 130 \times 27 \mathrm{~m}^{3}$, and consisted of 306,668 elements, is represented by 8 -noded brick elements (see Figure 2). In the ballast and embankment, the average element body size was about $0.375 \times 0.5 \times 0.25 \mathrm{~m}$. The rail and sleeper elements size was $0.05 \times 0.2 \times 0.05 \mathrm{~m}$ and 0.1 $\times 0.1 \times 0.15 \mathrm{~m}$, respectively. The whole system is divided into four single formulated substructures, that is, the track, ballast, embankment, and the ground, respectively. Ballast is placed on the top of embankment, supporting the rails and the sleepers. The fixed boundary was used in the bottom of the model. Infinite elements based on the previous work by Lysmer and Kuhlemeyer [41], and Kouroussis et al. [42] are used on the $X$ and $Z$ direction boundaries to represent the infinite boundary condition to absorb $S$ and $P$ waves. The nodes at the bottom boundary were fixed in every direction to simulate the bedrock. Both ends of the ground boundary were fixed in every direction in order to keep the ground in 


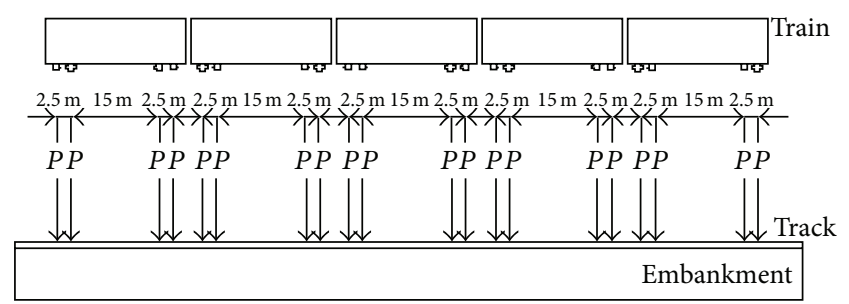

(a) Geometry and axle loads of five-carriage train

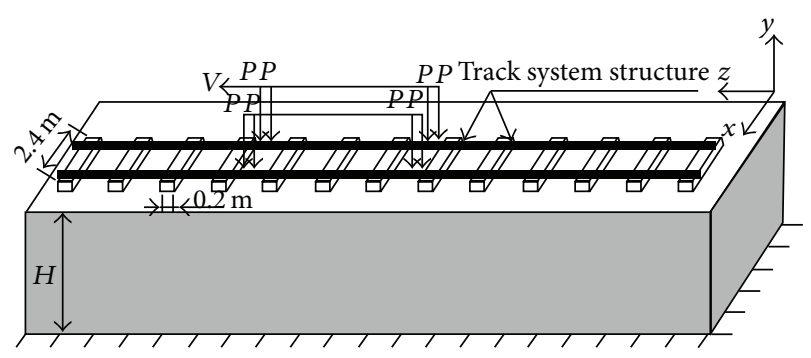

(b) Load mode with track structure

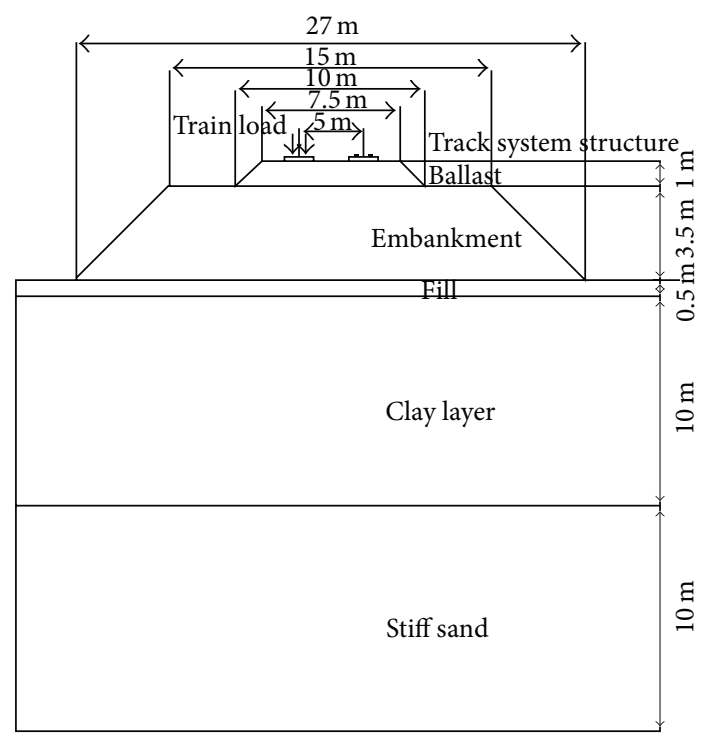

(c) Geometry of track-embankment-ground system

FIGURE 1: Load model and geometry of track-embankment-ground system.

place at the ends of the finite element model. The explicit central difference method [43] is used for time integration of the dynamic equilibrium equations. Program ABAQUS (Dassault Systems 2007) is used for finite element simulation, in which both the Lysmer-Kuhlemeyer (LK) infinite element and the explicit solver are available integrated.

A parametric analysis is carried on considering different rail, ballast, embankment, and ground soil properties. The calculated parameters of finite simulation were shown in Table 1. The track, ballast, embankment, and ground are assumed to be linear elastic. Rayleigh damping represents energy-dissipating mechanisms in the ground. The mass and stiffness proportional damping constants of ground are $\alpha=$ 1.2 and $\beta=0.0004$, which provide slight damping ratios of $2-4 \%$ in the frequency range of $3-50 \mathrm{~Hz}$. Some observation points shown in Figure 2(b) are selected for further study.

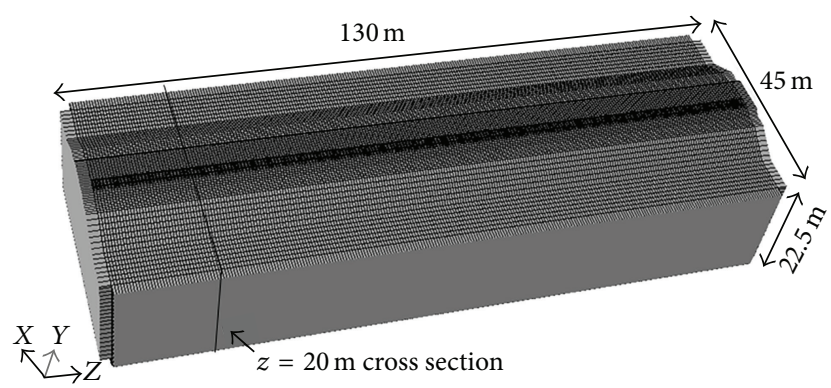

(a) Three dimensional view of the model

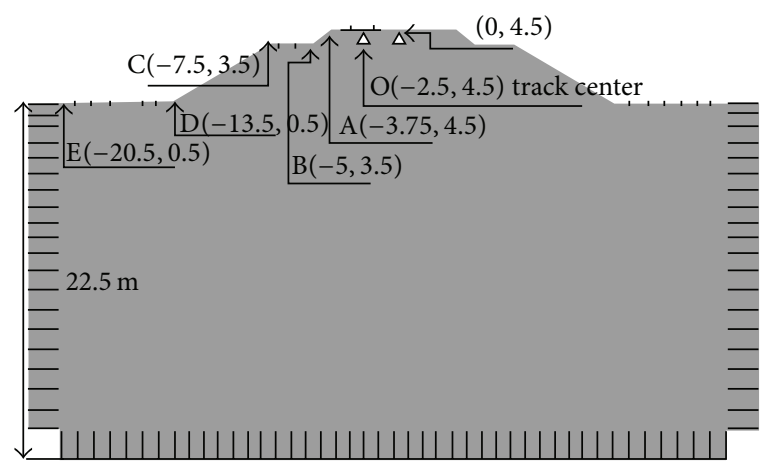

(b) Cross section of the mode $1(z=20 \mathrm{~m})$

FIGURE 2: Finite element mesh of the three-dimensional model.

TABLE 1: Material parameters of finite simulation.

\begin{tabular}{lccccc}
\hline Material & $E / \mathrm{Pa}$ & $\mu$ & $\rho / \mathrm{kg} / \mathrm{m}^{3}$ & $V_{S}$ & $V_{R}$ \\
\hline Ballast & $3.89 E+8$ & 0.3 & 2200 & 260.8 & 241.9 \\
Embankment & $2.5 E+8$ & 0.3 & 1800 & 231.1 & 214.4 \\
Fill & $9.075 E+7$ & 0.3 & 1800 & 138.7 & 128.6 \\
Clay & $25.36 E+86$ & 0.35 & 1600 & 76.6 & 71.6 \\
Stiff sand & $1 E+8$ & 0.3 & 1800 & 146.2 & 135.6 \\
Rail & $2.1 E+11$ & 0.17 & 7800 & 3392 & 3074.2 \\
Sleeper & $2 E+10$ & 0.2 & 2500 & 1826 & 1664.5 \\
\hline
\end{tabular}

Note: $E$ is Young's modulus; $\rho$ is density; $\mu$ is Poisson's ratio; $V_{S}$ and $V_{R}$ are shear and Rayleigh wave speeds, respectively.

\section{Dynamic Responses of Track-Embankment- Ground System}

In order to investigate the dynamic characteristics of the track-embankment-ground system, the dynamic response due to five carriages travelling at different speeds was analyzed.

3.1. Dynamic Response of Track Structure. Figure 3 shows dynamic responses along the rail at time $1.54 \mathrm{~s}$ for five carriages travelling at a speed of $80 \mathrm{~m} / \mathrm{s}$. The Figure shows that the peaks of the vertical stress along the rail appear at the position where the train load is applied, the values of which are around 1.7 MPa. The peaks of vertical displacement roughly coincide with the instantaneous position of the moving loading unit, of which the value is around $0.00117 \mathrm{~m}$. 


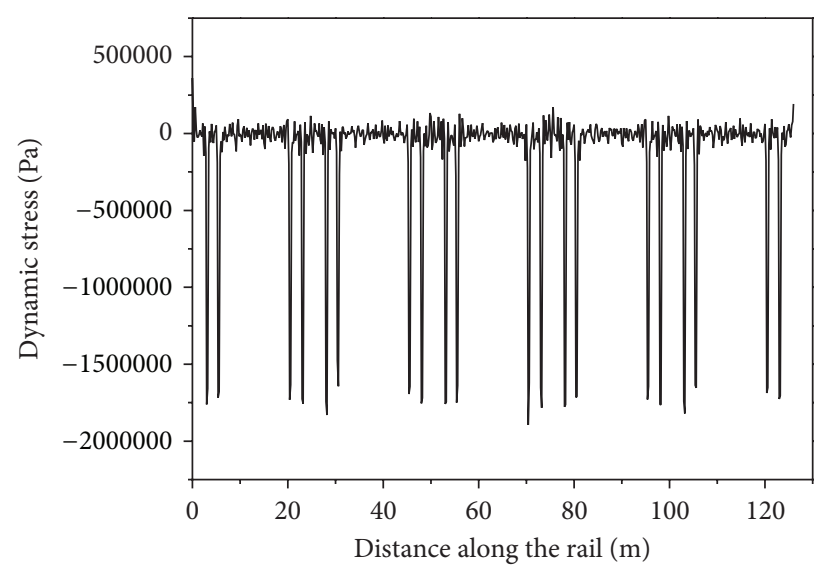

- Stress at rail top

(a) Dynamic vertical stress

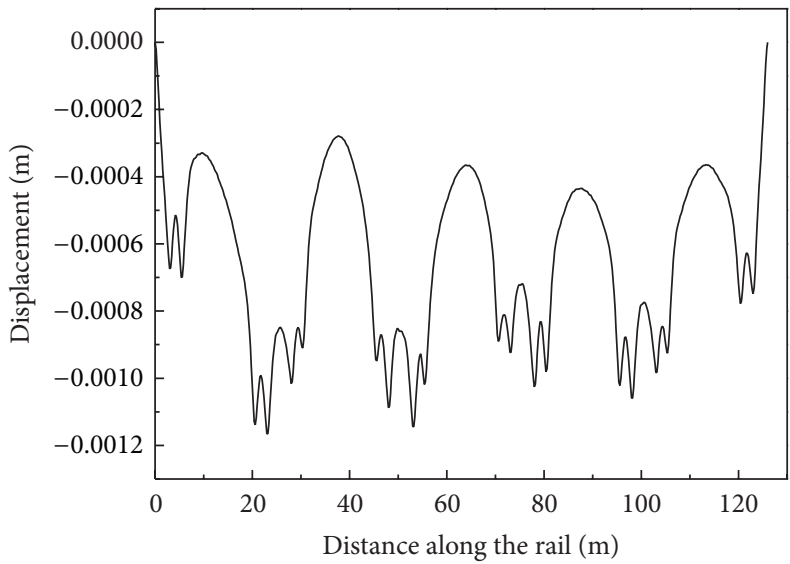

_ Displacement at rail top

(b) Displacement

Figure 3: Dynamic responses along the rail top at time $1.54 \mathrm{~s}$ for five carriages travelling at a speed of $80 \mathrm{~m} / \mathrm{s}$.

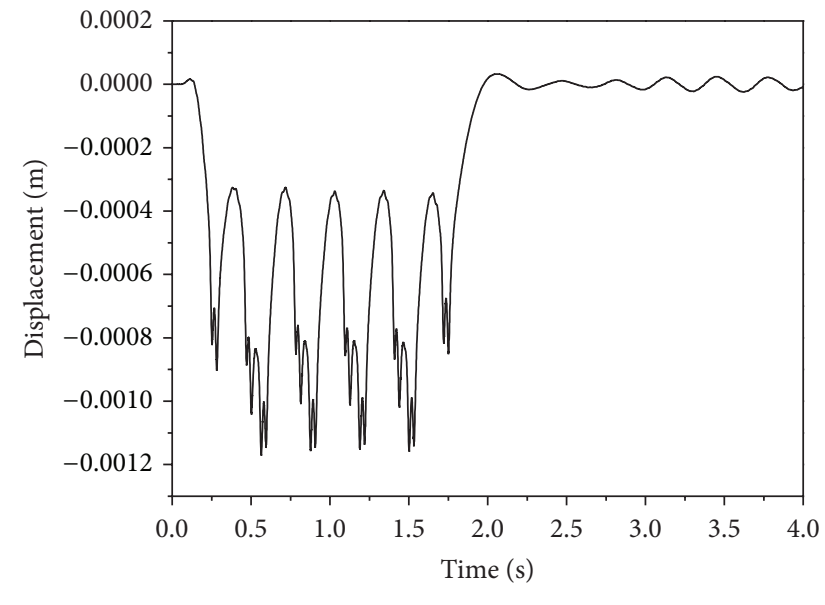

$-80 \mathrm{U} 2$

(a) Time history of the vertical displacement

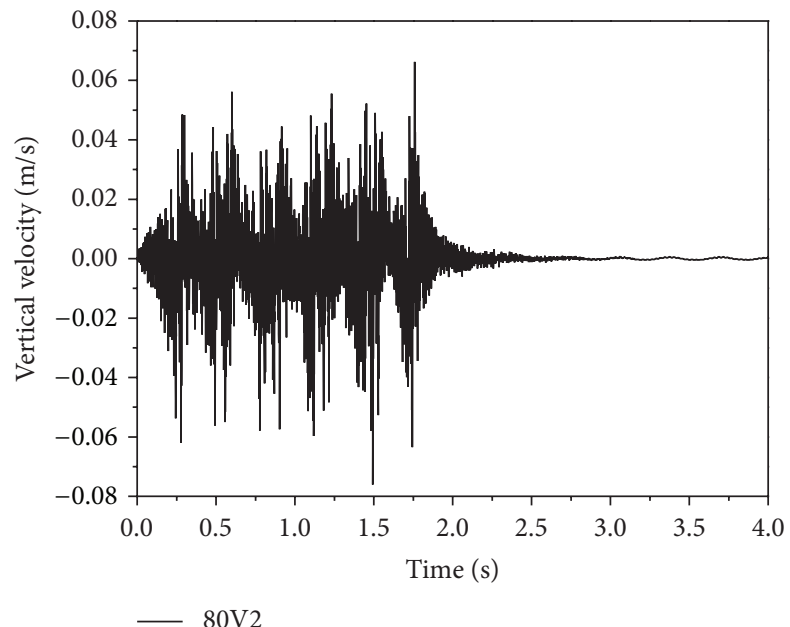

(b) Vertical velocity

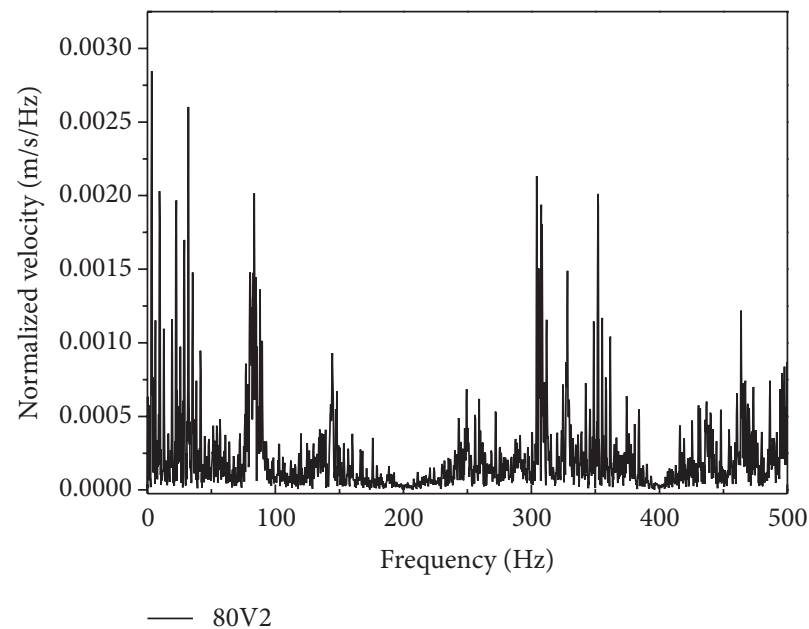

(c) Frequency content of the vertical velocity

FIgURE 4: Curves of dynamic response at the rail top for five carriages travelling at $v=80 \mathrm{~m} / \mathrm{s}(288 \mathrm{~km} / \mathrm{h})$. 


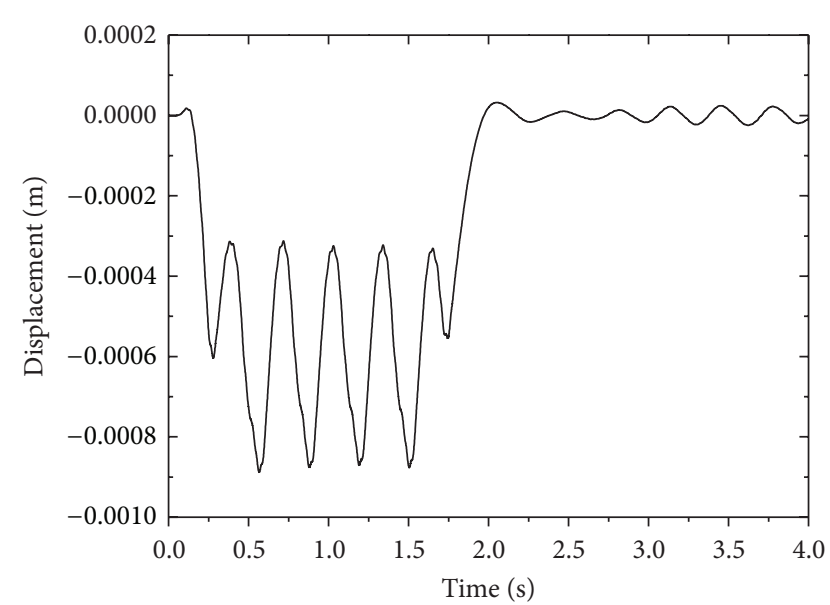

(a) $\mathrm{A}, 1.25 \mathrm{~m}$

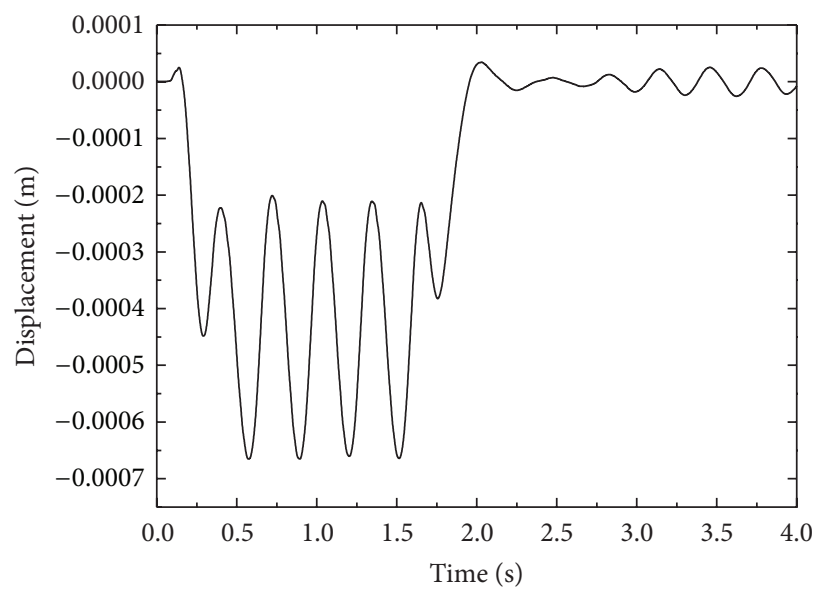

(c) $\mathrm{C}, 5 \mathrm{~m}$

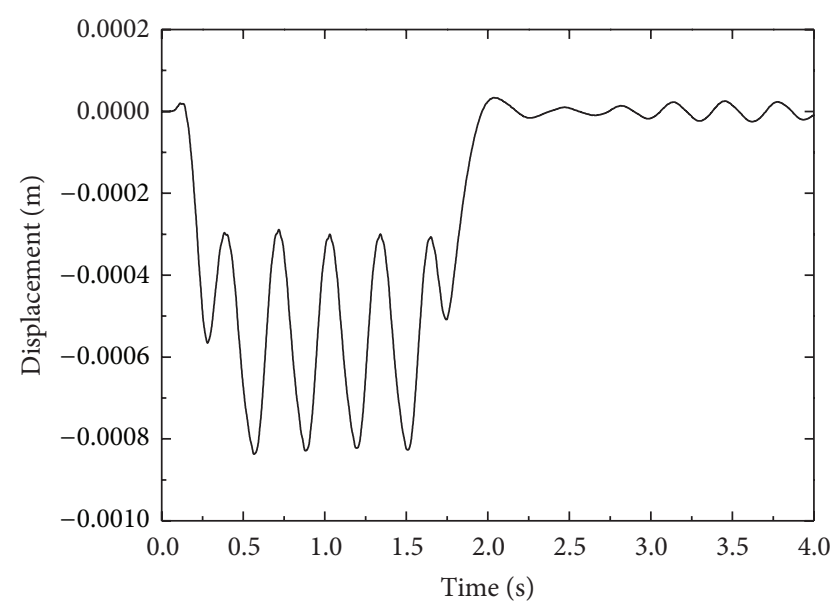

(b) $B, 2.5 \mathrm{~m}$

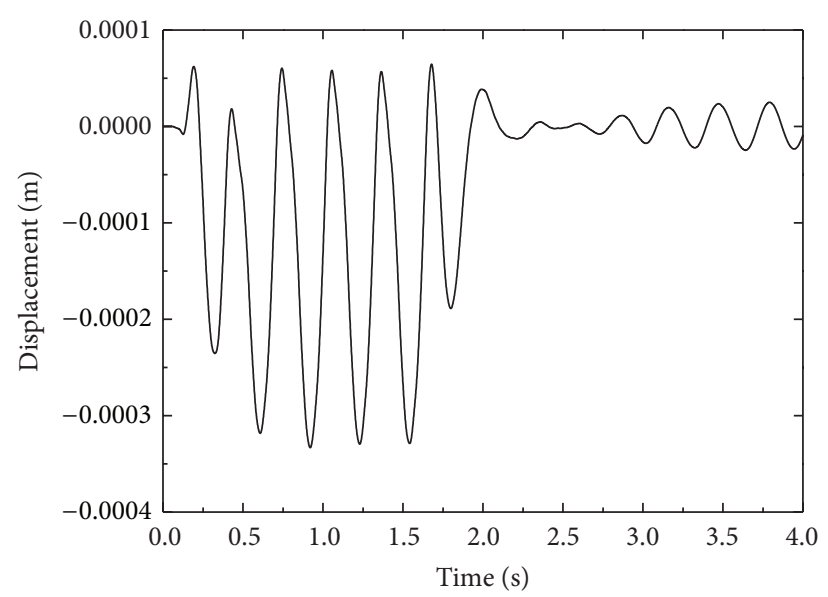

(d) $\mathrm{D}, 11 \mathrm{~m}$

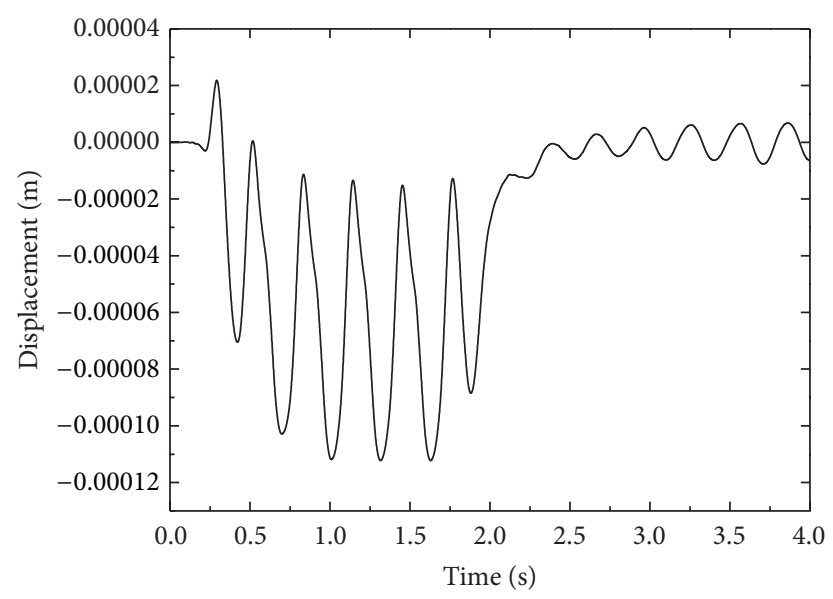

(e) E, $17.5 \mathrm{~m}$

FIgURE 5: The time history of the vertical displacement at selected points at different distances from the track center.

Figure 4 shows the time history of the vertical displacement, velocity, and the frequency content of the vertical velocity at the rail for five carriages travelling at $v=80 \mathrm{~m} / \mathrm{s}$ $(288 \mathrm{~km} / \mathrm{h})$. In Figure 4(a), it can be seen that the peaks of vertical displacement at rail appear as the train loads passing by correspondingly. The maximum displacement induced by the following carriage of train loads is $0.00117 \mathrm{~m}$, which is larger than that induced by the first sets of loads for the reason of dynamic superposition effect of moving loads. In Figure 4(b), the maximum value of vertical velocity at the 


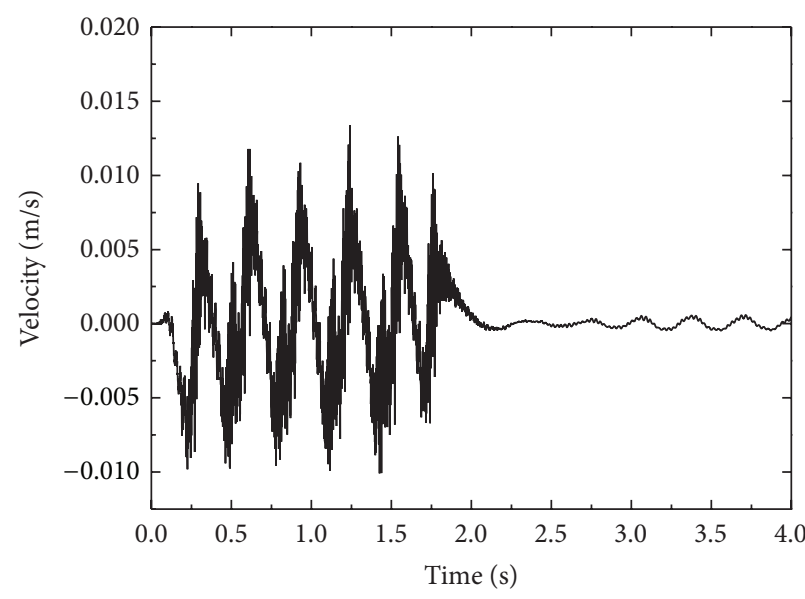

(a) A, $1.25 \mathrm{~m}$

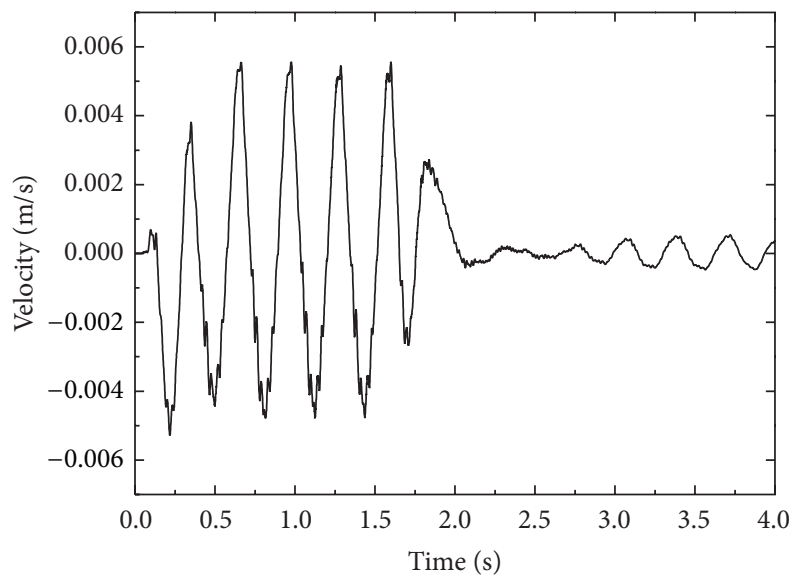

(c) $\mathrm{C}, 5 \mathrm{~m}$

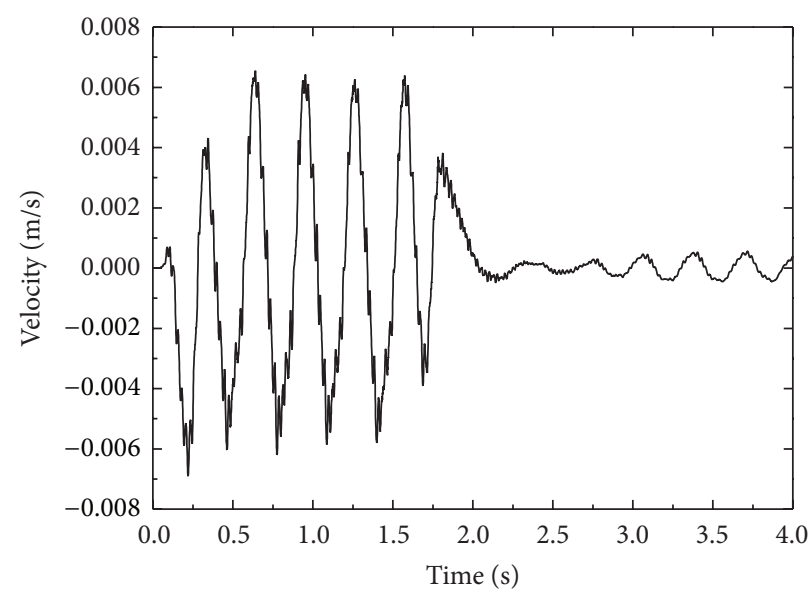

(b) B, $2.5 \mathrm{~m}$

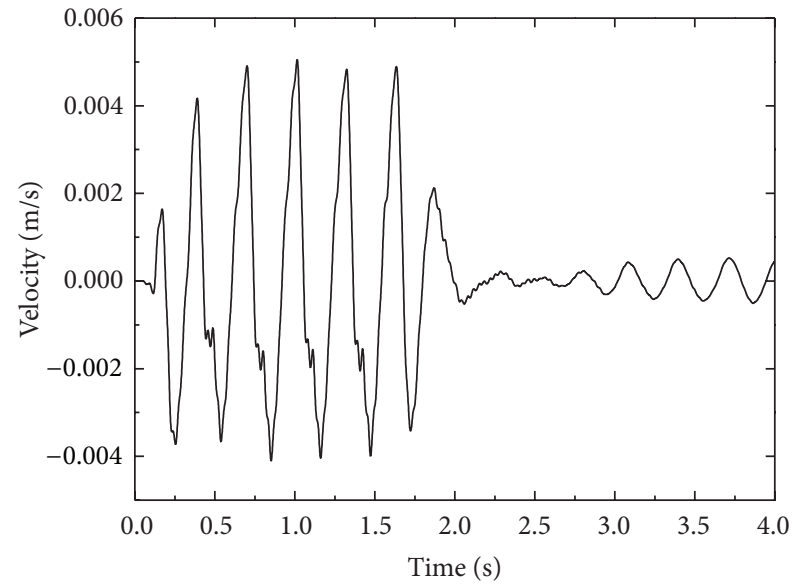

(d) $\mathrm{D}, 11 \mathrm{~m}$

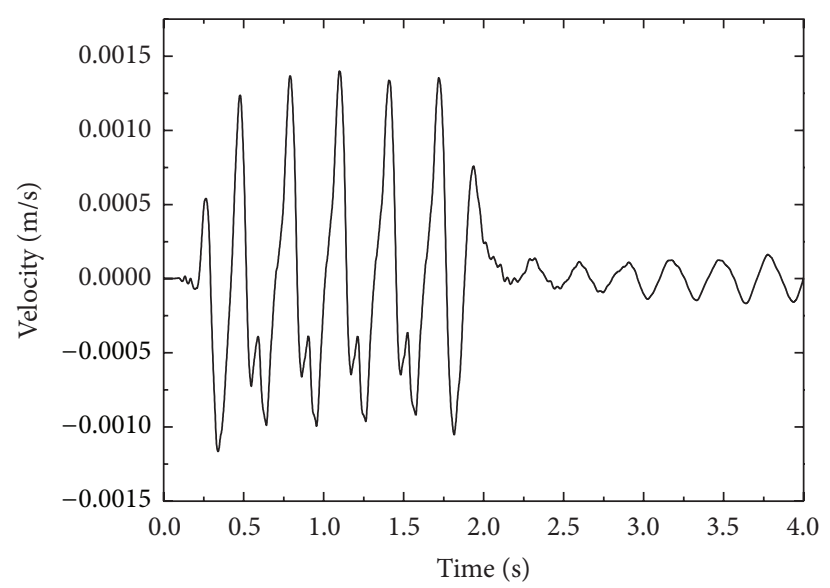

(e) E, $17.5 \mathrm{~m}$

FIGURE 6: The time history of the vertical velocity at selected points at different distances from the track center.

rail surface is $0.075 \mathrm{~m} / \mathrm{s}$, which decreases as the train load goes away. As the interbogie spacing $L_{b}=2.5 \mathrm{~m}$, the axle spacing $L_{a}=25 \mathrm{~m}$, for speeds $v=80 \mathrm{~m} / \mathrm{s}$. The bogie passing frequency $f_{b}=v / L_{b}=3.2 \mathrm{~Hz}$ (low frequency) and the axle load passage frequency $f_{a}=v / L_{a}=32 \mathrm{~Hz}$. In Figure 4(c), the frequency content of the vertical velocity at the rail shows peaks both in the bogie passing frequency $3.2 \mathrm{~Hz}$ and the axle passing frequency $32 \mathrm{~Hz}$. The peak velocity appears in the low, medium, and high frequency area. Therefore, the rail response shows the contributions of the resonance frequency of the track model on the dynamic response of track-ballastembankment-ground system. Similar results are presented 


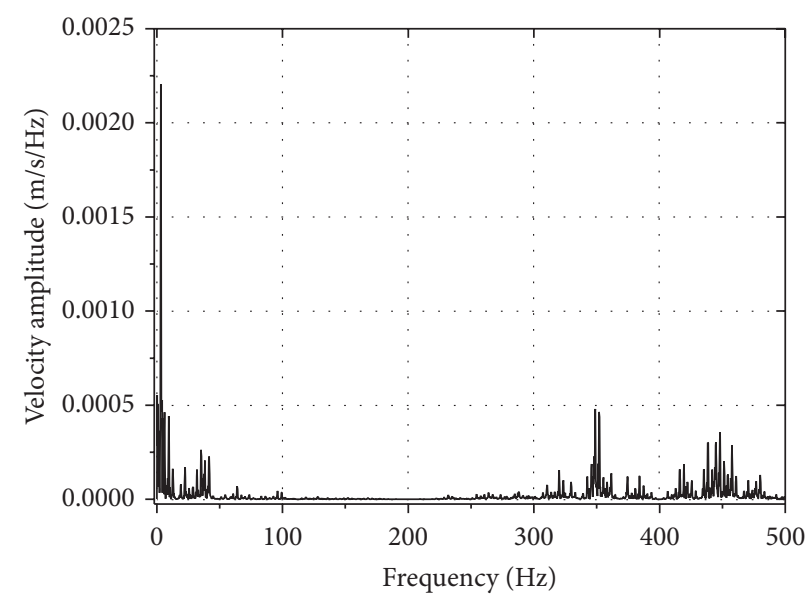

(a) A, $1.25 \mathrm{~m}$

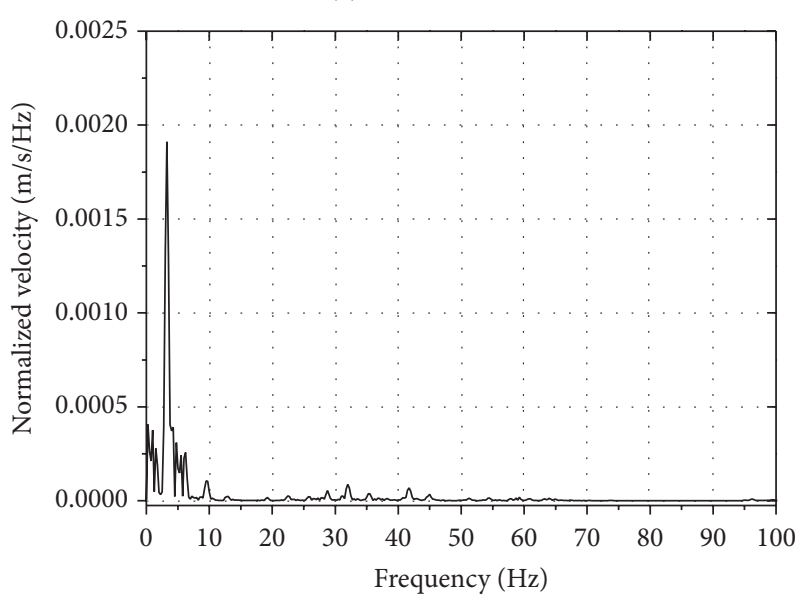

(c) C, $5 \mathrm{~m}$

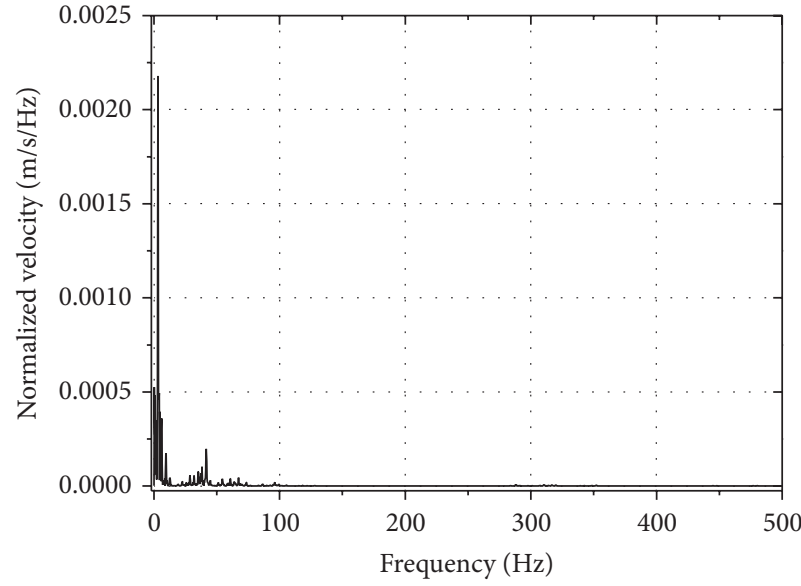

(b) B, $2.5 \mathrm{~m}$

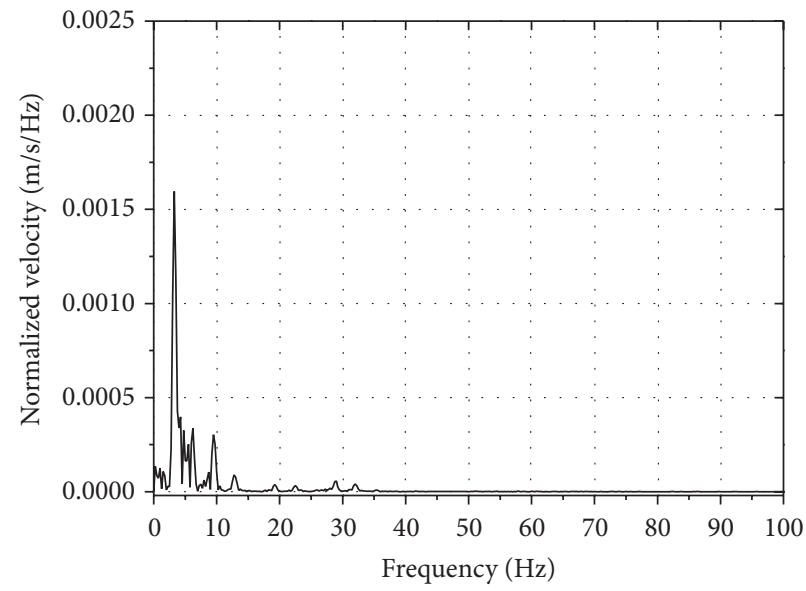

(d) $\mathrm{D}, 11 \mathrm{~m}$

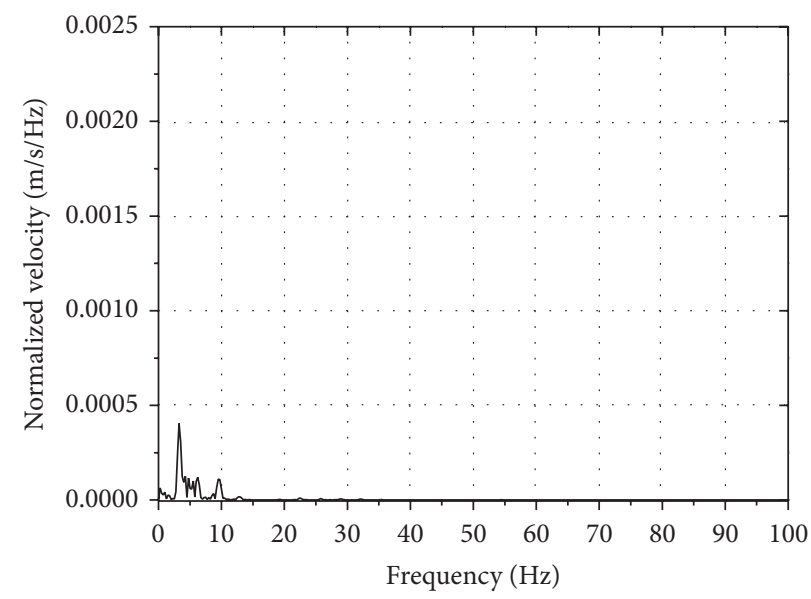

(e) E, $17.5 \mathrm{~m}$

FIGURE 7: The frequency contents of the vertical velocity at selected points at different distances from the track center: $v=80 \mathrm{~m} / \mathrm{s}$.

by Kaewunruen and Remennikov [39]. The measured time history and frequency content of velocity at the sleeper during the passage of an AVE-Alstom HST travelling at a speed, $v=298 \mathrm{~km} / \mathrm{h}$ on Track1, show peaks in the bogie passing frequency, $f_{b}=v / L_{b}=4.43 \mathrm{~Hz}$, and related high-order harmonic frequencies and in the axle passing frequency, $f_{a}=$ $v / L_{a}=27.59 \mathrm{~Hz}$, as well.
3.2. Dynamic Response of Embankment-Ground System. The time histories of the vertical displacement at selected points A, B, C, D, and E (see Figure 2) at different distances from the track center are shown in Figure 5. It is noted that the vertical displacement at rail is larger than that at embankment, and the displacement peaks coincide with instantaneous position of the train load. However, the displacement peaks at other 


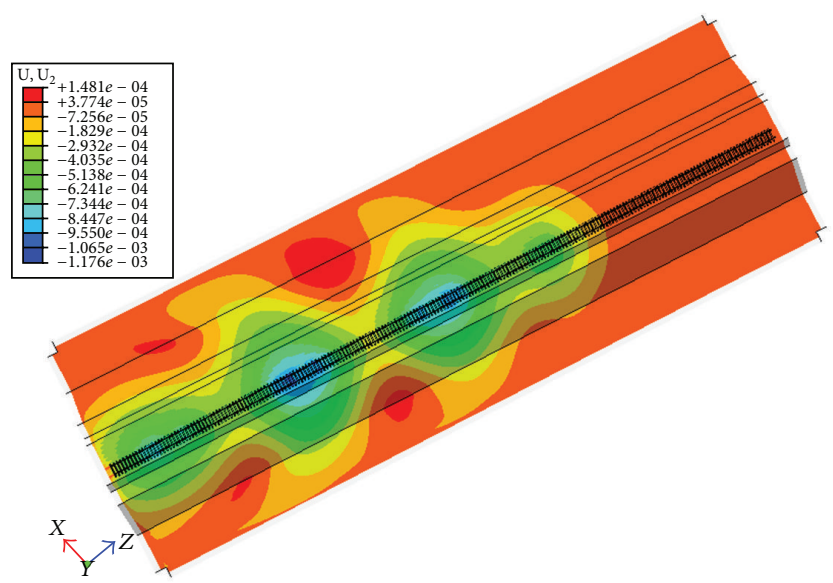

(a) $1.04 \mathrm{~s}$

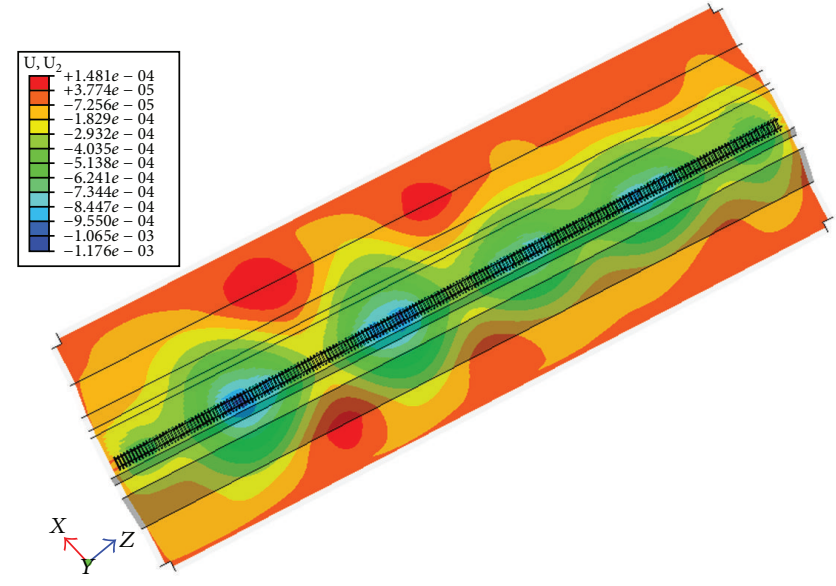

(b) $1.54 \mathrm{~s}$

FIGURE 8: Typical contour plots of vertical displacement at different times for the speed $v=80 \mathrm{~m} / \mathrm{s}$.

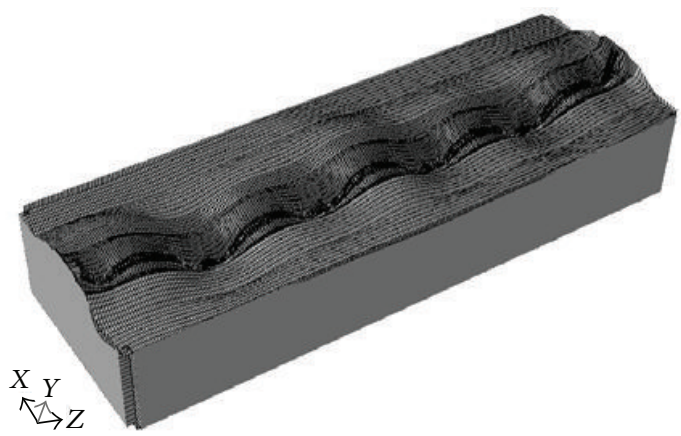

(a) $v=60 \mathrm{~m} / \mathrm{s}, t=2.06 \mathrm{~s}$

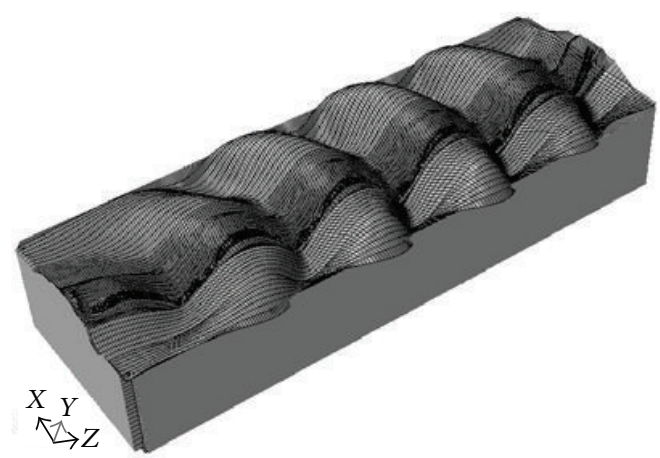

(c) $v=100 \mathrm{~m} / \mathrm{s}, t=1.22 \mathrm{~s}$

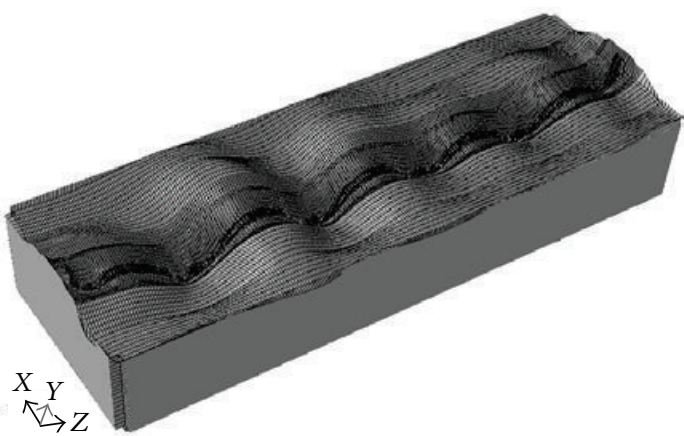

(b) $v=80 \mathrm{~m} / \mathrm{s}, t=1.54 \mathrm{~s}$

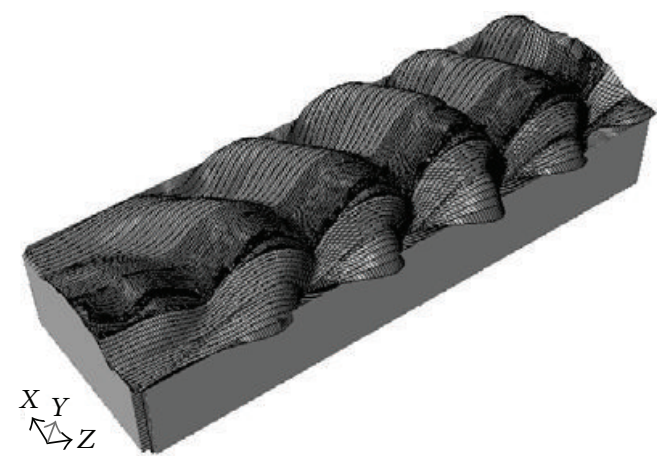

(d) $v=120 \mathrm{~m} / \mathrm{s}, t=1.02 \mathrm{~s}$

FIGURE 9: Full views of deformed meshes of displacement magnified with a factor of 15000 from finite analysis of train-induced ground vibrations for different train speeds travelling from left to right.

points appear to gradually get more out of phase and decrease as the distance from the track center increases. Thus, the vibration waves spread out from the track center and decrease for the vibration attenuation, which shows that the threedimensional model is suitable for simulating the dynamic response of track-embankment-ground system.

Figure 6 shows the time histories of vertical velocity at selected points for train travelling with a speed of $80 \mathrm{~m} / \mathrm{s}$. It can be seen from the figure that with increase of distance to track center the velocity level of embankment and ground decreases gradually. The peak value of velocity at point A is $0.013 \mathrm{~m} / \mathrm{s}$, which decreases gradually with the increase of distance. It reaches $0.0013 \mathrm{~m} / \mathrm{s}$ at $17.5 \mathrm{~m}$. As mentioned previously, the dynamic response attenuations quickly within $17.5 \mathrm{~m}$. According to the spectrum of velocity response in Figure 7, the peak amplitude points at different distances from the track center appear around different frequency areas, and the peak values of velocity are also different. The 


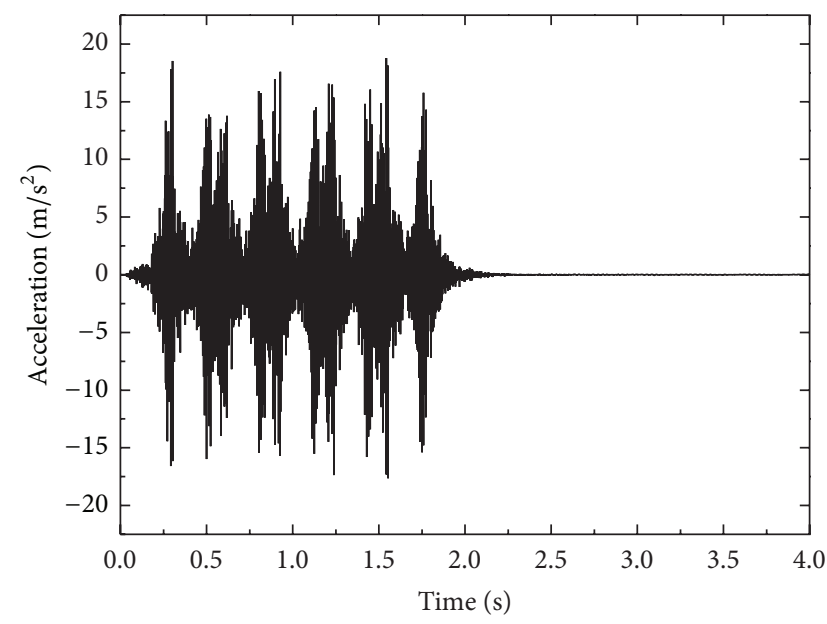

(a) A, $1.25 \mathrm{~m}$

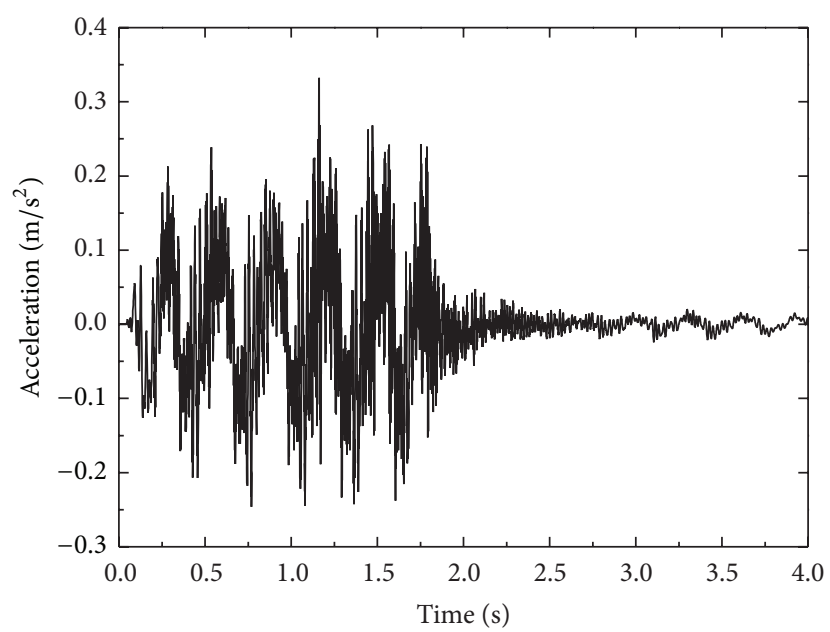

(c) C, $5 \mathrm{~m}$

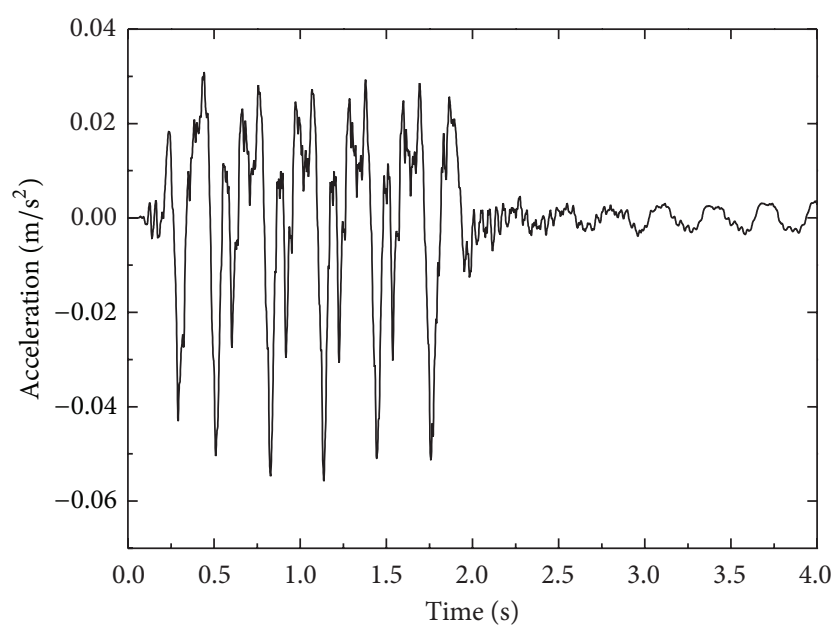

(e) E, $17.5 \mathrm{~m}$

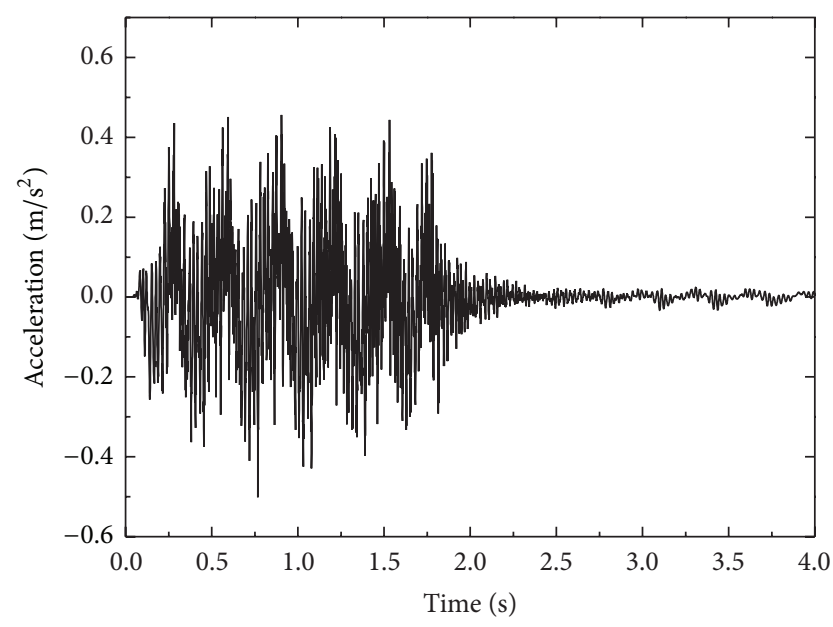

(b) B, $2.5 \mathrm{~m}$

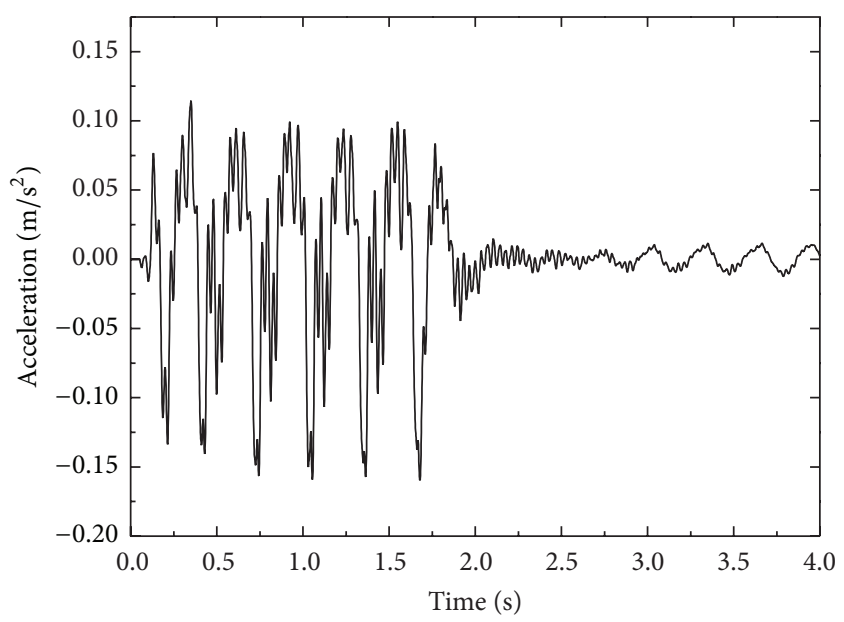

(d) $\mathrm{D}, 11 \mathrm{~m}$

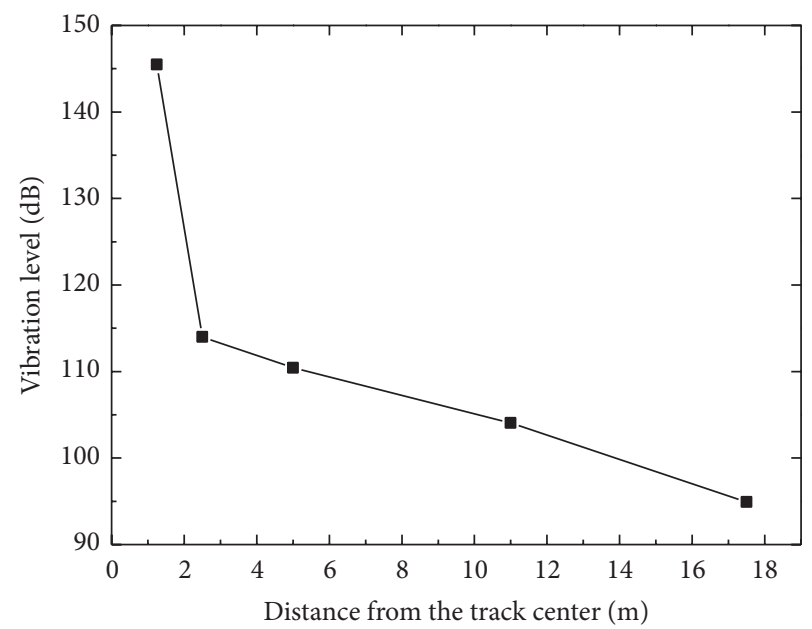

(f) vibration level curve

FIGURE 10: Time history of acceleration at selected points at different distances from the track center: $v=80 \mathrm{~m} / \mathrm{s}((\mathrm{a})-(\mathrm{e}))$ and (f) vibration level curve. 


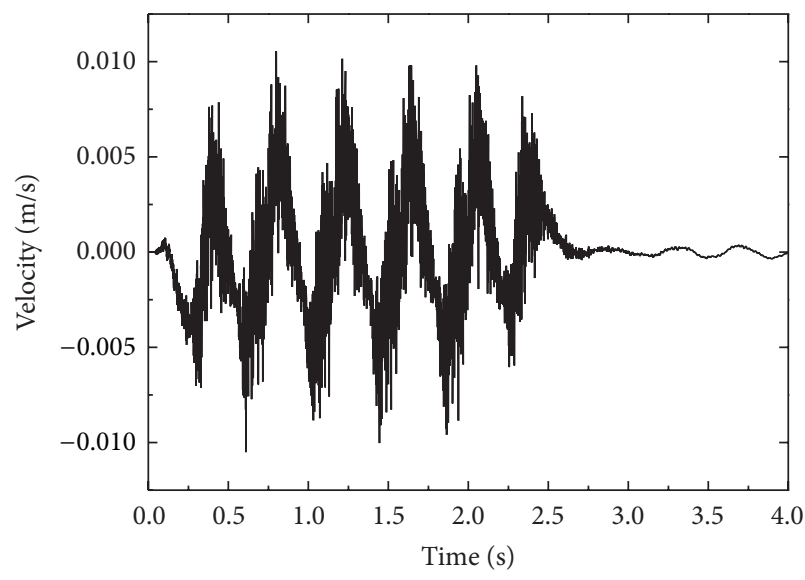

(a) $v=60 \mathrm{~m} / \mathrm{s}$

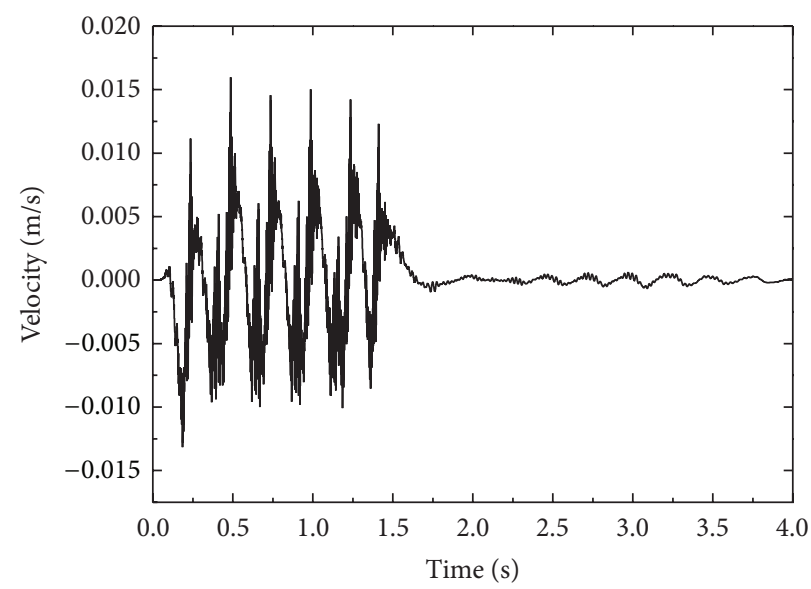

(c) $v=100 \mathrm{~m} / \mathrm{s}$

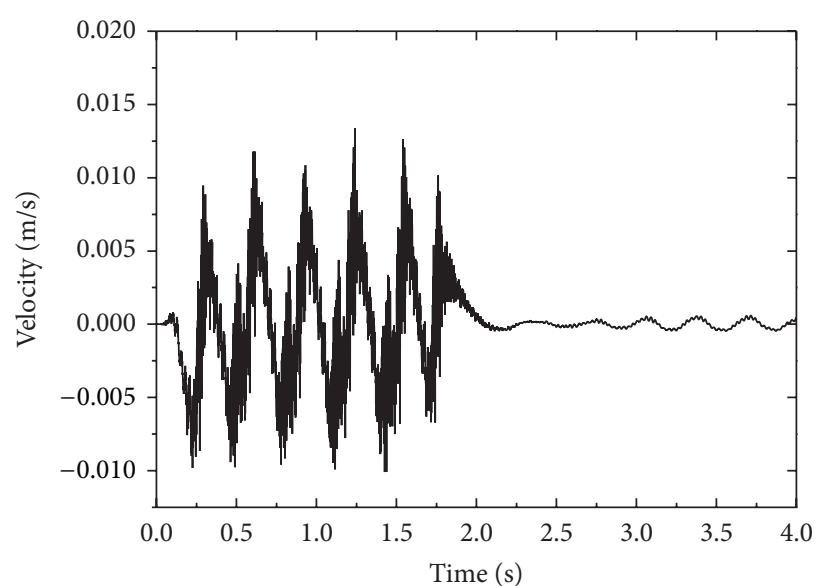

(b) $v=80 \mathrm{~m} / \mathrm{s}$

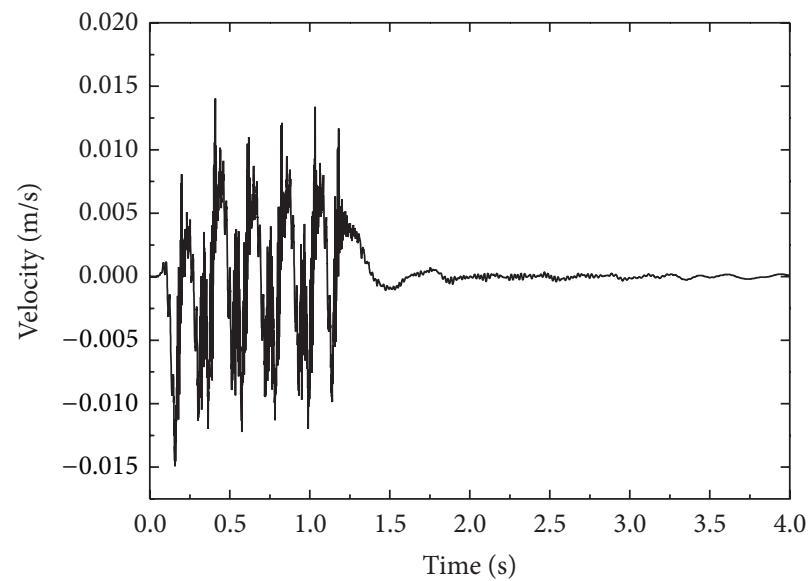

(d) $v=120 \mathrm{~m} / \mathrm{s}$

FIGURE 11: Time history of vertical velocity at a point $1.25 \mathrm{~m}$ from the track center for different train speeds.

spectrum of velocity response consisted of low, medium, and high frequencies, showing the vibration character and level near the excitation source (shown in Figure 7(a)), as is shown in Figures 7(c), 7(d), and 7(e). The vibration velocity amplitude decreases with the decrease of distance from the track center, and the peaks move toward low frequency, appearing at frequency below $10 \mathrm{~Hz}$.

Figure 8 shows the contour plots of the displacement for a train speed of $80 \mathrm{~m} / \mathrm{s}$ at $1.04 \mathrm{~s}$ and $1.54 \mathrm{~s}$. The moving loads enter the model at the left end and travel $130 \mathrm{~m}$ on the rail before leaving the model at the right end. The wave fronts of the induced stress waves were developed when they entered the finite element model. Instead, the wave fronts are created as soon as the moving loads, from which they originate, enter the model. The development of the wave fronts depends on the geometrical spreading of the waves and the propagation velocities of the different waves. It can here be observed that the wave fronts at the head consisted of circles at $1.04 \mathrm{~s}$, and the circles induced by the latter loads spread out as the train passes by. The maximum downward displacements roughly coincide with the instantaneous position of the train loads. A dynamic superposition effect appears on ballast surface when the following wheel loads passing by, so the maximum displacement on the ballast surface for the first set of loads is lower than that for the following sets of loads. At the time of $1.54 \mathrm{~s}$ the wave fronts have become more developed and spread out from the source.

Here, for illustration purpose, the full views of deformed meshes of displacement for train speeds of $60 \mathrm{~m} / \mathrm{s}, 80 \mathrm{~m} / \mathrm{s}$, $100 \mathrm{~m} / \mathrm{s}$, and $120 \mathrm{~m} / \mathrm{s}$ are shown in Figure 9 . For train speeds under subcritical ranges $(v=60 \mathrm{~m} / \mathrm{s})$ lower than the propagation velocity of the soft soil ( $71.6 \mathrm{~m} / \mathrm{s}$ see Figure 9(a)), the induced wave fronts were perpendicular to the loads, and the deformation pattern is roughly symmetrical with each bogie imprint, which is similar to the displacement distribution for quasi-static case.

When the train speed is supercritical (see Figures 9(b), 9(c), and 9(d)), the induced wave fronts here show a ploughshaped behaviour following the loads, which are no longer perpendicular to the moving loads. Instead, they show plough shaped behaviour similar to the wash from a motor boat. The phase angles between the wave fronts and the loads moving direction decrease with the increase of moving speed. Behind the train, a series of trailing oscillations are observed. Similar 


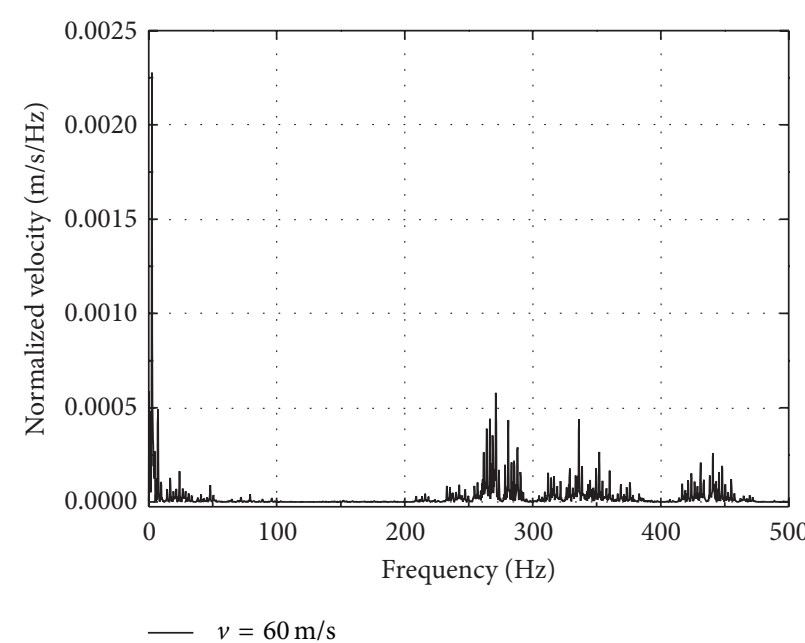

(a)

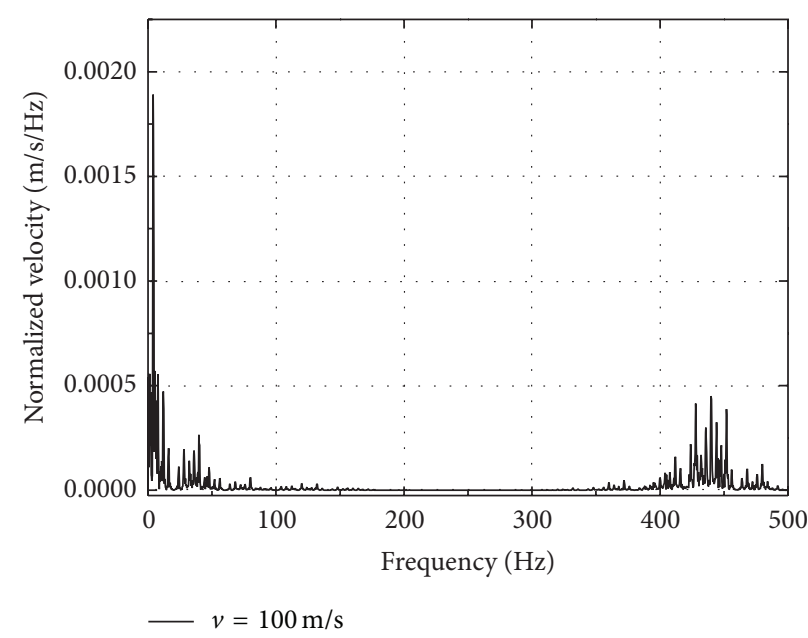

(c)

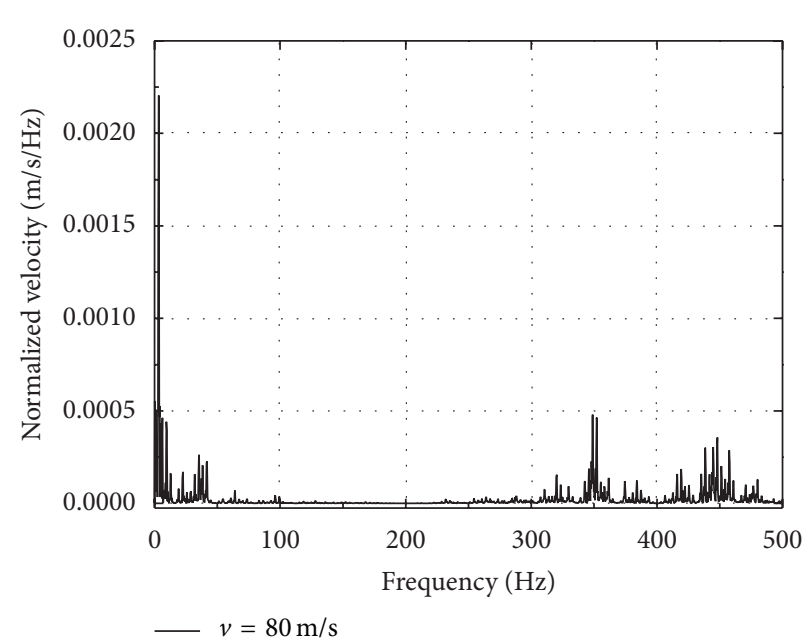

(b)

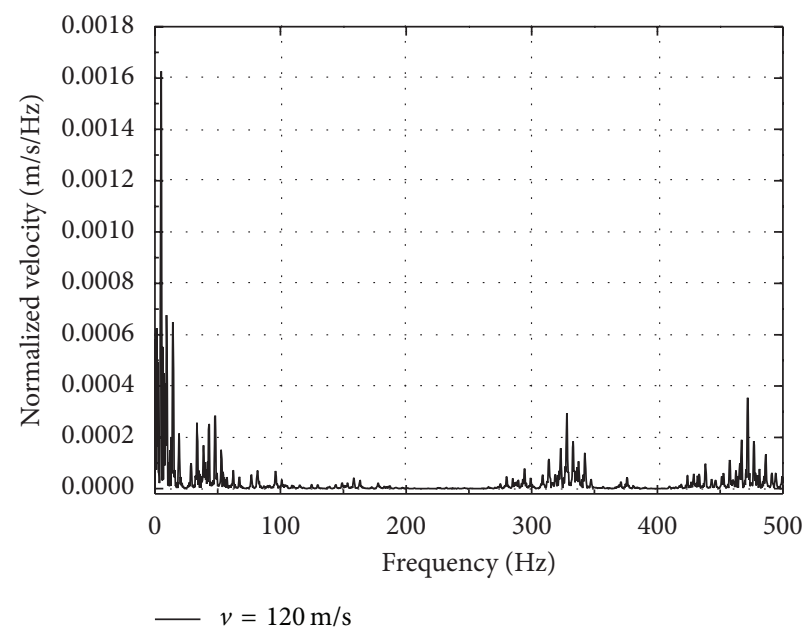

(d)

FIGURE 12: Frequency contents of vertical velocity at point $1.25 \mathrm{~m}$ from the track center for different train speeds.

results, in the case of three- dimensional model subjected to moving point loads, can be found in $[34,44]$. Moreover, the motion orbits calculated from the three-dimensional analyses indicate that the Rayleigh wave is the dominating wave type outside the embankment [44]. At the critical speed case, the track critical velocity is slightly higher than the Rayleigh wave velocity due to the ballast acting as a beam [10]. Full Rayleigh wave effects therefore occur at speeds slightly higher than the Rayleigh ground wave speed.

Time histories of acceleration at points A, B, C, D, and $\mathrm{E}$ and the vibration level curve are shown in Figure 10. The wheel effect and double peak appear in time history of vertical acceleration at distance $1.25 \mathrm{~m}$ from the track center under the train load. At this observation point near the excitation source, the maximum acceleration is approximately $18 \mathrm{~m} / \mathrm{s}^{2}$. The acceleration amplitudes of the points located on the embankment and ground surface decrease with the increase of distance from the track center, which show attenuation according to the geometrical damping and internal damping of the soil. At a distance $17.5 \mathrm{~m}$ from the track center, the maximum acceleration is approximately $0.055 \mathrm{~m} / \mathrm{s}^{2}$. The vibration measurements were taken out on embankment at Shinkansen in Japan, which show that the maximum acceleration appears around $5 \sim 20 \mathrm{~m} / \mathrm{s}^{2}$. The vibration level limit in Japan is $90 \mathrm{~dB}$. The formula [45] of acceleration vibration level adopted here is $\mathrm{VAL}=20 \log \left(a_{\mathrm{rms}} / a_{0}\right)$, where VAL is acceleration vibration level $(\mathrm{dB}), a_{\mathrm{rms}}$ is effective value of vibration acceleration $\left(\mathrm{m} / \mathrm{s}^{2}\right), a_{0}$ is reference acceleration, and its value is $1 e-6 \mathrm{~m} / \mathrm{s}^{2}$ according to ISO Standard [46]. The vibration levels of these points corresponding with distance from the track center are shown in Figure 8, decrease with the increase of distance from the track center. It can be seen that the vibration level at a distance $17.5 \mathrm{~m}$ is $94 \mathrm{~dB}$ close to the value limit $90 \mathrm{~dB}$ in Japan's vibration standard.

\section{Parametric Study}

Due to high speed train passage, track-ballast-embankmentground properties play an important role in the ground 


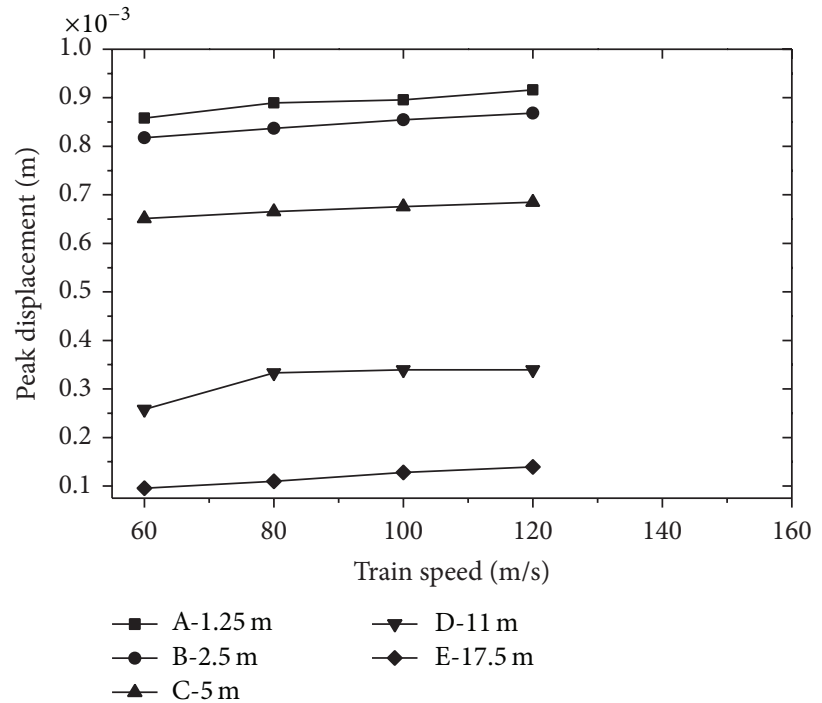

(a) Peak displacement

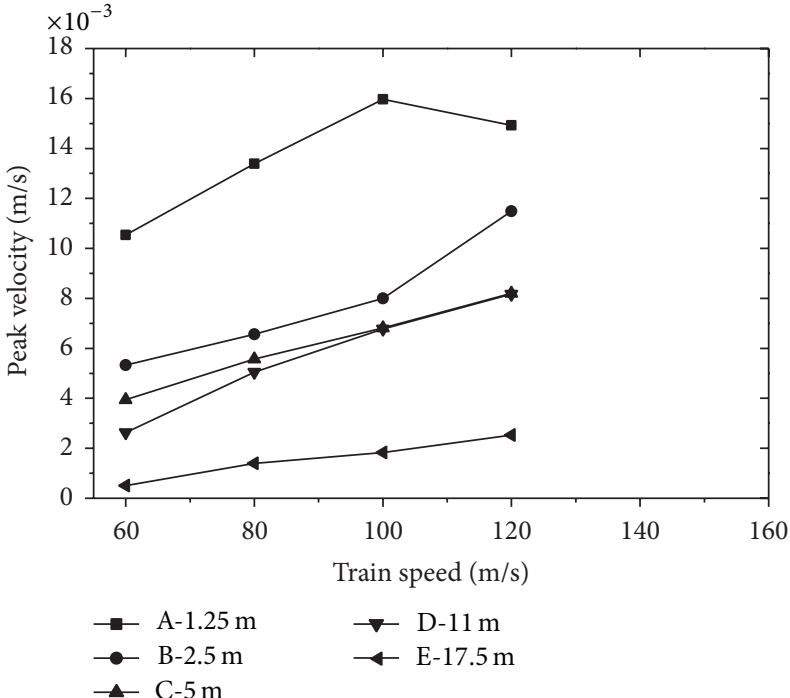

(b) Peak velocity

FIGURE 13: Peak displacement and velocity at selected points for different train speeds.

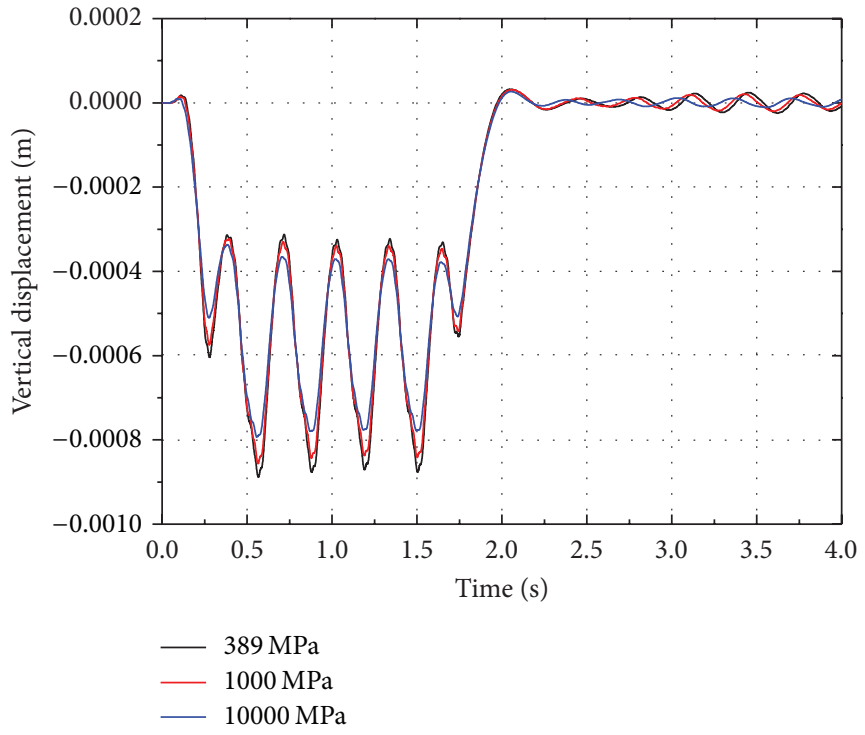

(a) Vertical displacement

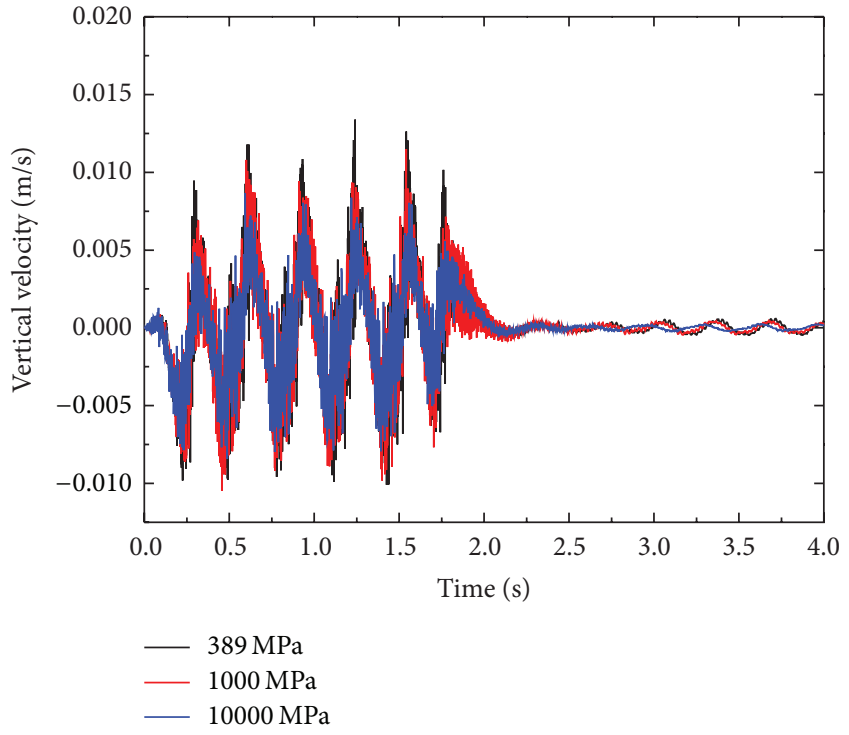

(b) Vertical velocity

Figure 14: Time histories of dynamic response at point $1.25 \mathrm{~m}$ from the track center for different ballast modulus.

vibration. Their effects can be evaluated by representing the actual ballast and embankment properties using the FEM presented in this paper. In order to investigate this effect, some different numerical simulations are carried out. The effects of train axle load and train speed are also discussed in the following section.

4.1. Effect of Train Speed. This section conducts a preliminary study of the train load fundamental passing frequency effect on track-ballast-embankment-ground vibration under four train speeds $(60 \mathrm{~m} / \mathrm{s}, 80 \mathrm{~m} / \mathrm{s}, 100 \mathrm{~m} / \mathrm{s}$, and $120 \mathrm{~m} / \mathrm{s}$, resp.). Since the ground Rayleigh wave speed is $C_{R}=71.6 \mathrm{~m} / \mathrm{s}$, these speeds are in the subcritical, critical, and supercritical ranges, respectively. Figure 11 shows the time history of vertical velocity at a point $1.25 \mathrm{~m}$ from the track center for above train speeds. By referring to the time history, one can observe the passing of every bogie and axle of the train. It is noted that the vibration amplitude of vertical velocity increases with the train speed but tends to fall when the train speed (for $v=120 \mathrm{~m} / \mathrm{s}$ see Figure 11(d)) comes near the fill Rayleigh wave speed $C_{R}=128.6 \mathrm{~m} / \mathrm{s}$.

Frequency spectra for the vertical velocity are obtained using the Fourier transform. Frequency spectra are shown in Figure 12 at point $1.25 \mathrm{~m}$ from the track center for different 


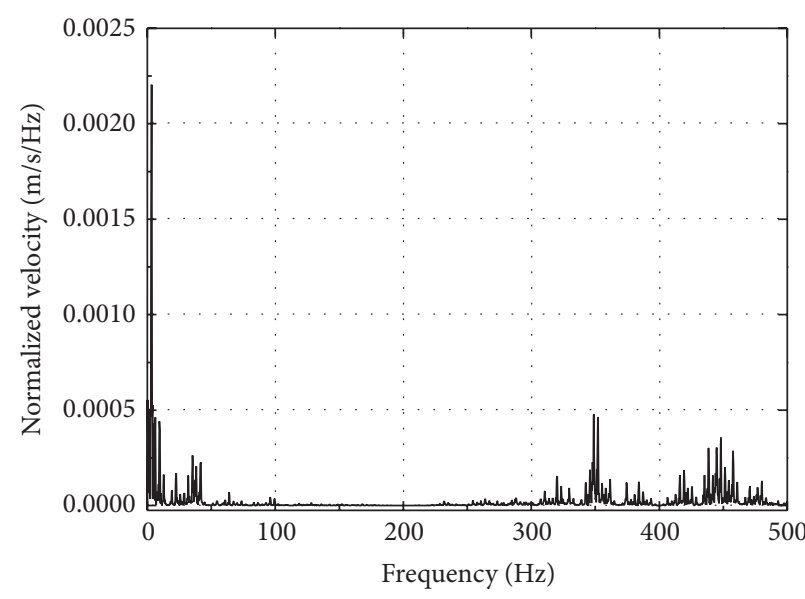

$-389 \mathrm{MPa}$

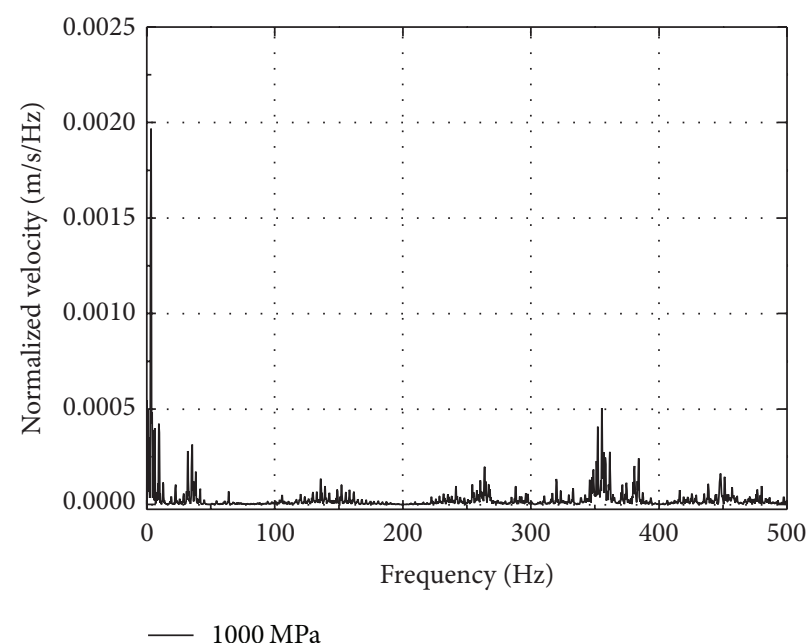

(b)

(a)

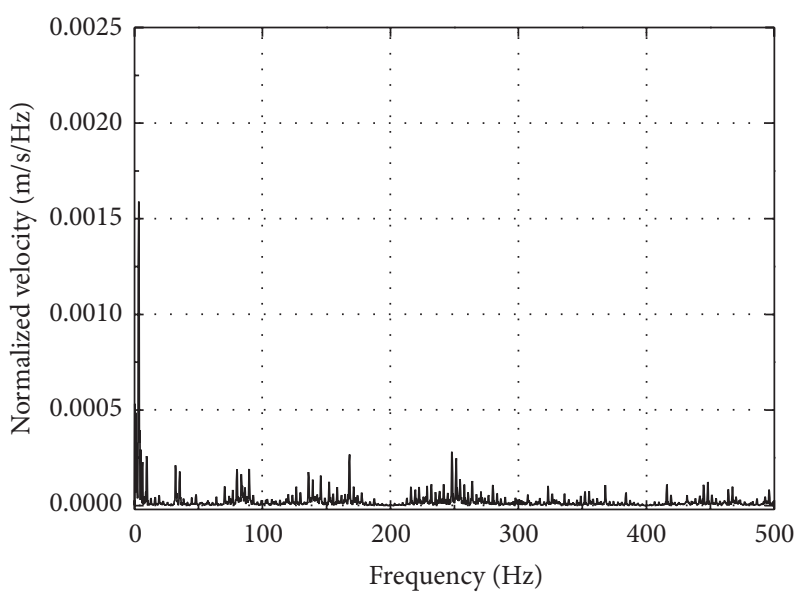

$-10000 \mathrm{MPa}$

(c)

Figure 15: The velocity amplitude of Fourier spectrum for different ballast modulus.

speeds. As the interbogie spacing $L_{b}=2.5 \mathrm{~m}$, the axle spacing $L_{a}=25 \mathrm{~m}$, and the bogie passing frequency $f_{b}=v / L_{b}$, the axle load passage frequency $f_{a}=v / L_{a}$. It can be observed from these figures that the frequency contents show peaks in the bogie passing frequency $2.4 \mathrm{~Hz}$, $3.2 \mathrm{~Hz}, 4 \mathrm{~Hz}$, and $4.8 \mathrm{~Hz}$ (low frequency) and in the axle load passage frequency $24 \mathrm{~Hz}, 32 \mathrm{~Hz}, 40 \mathrm{~Hz}$, and $48 \mathrm{~Hz}$ for speeds mentioned above. Also peaks are noted that are associated with rail and sleeper vibration character in the high frequency range larger than $200 \mathrm{~Hz}$. The velocity peaks tend to move toward high frequency range with the increase of train speeds, close to $450 \mathrm{~Hz}$ for train speed $80 \mathrm{~m} / \mathrm{s}$ in Figure 12(b).

Figure 13 shows the effect of train speed on peak displacement and velocity on the ballast-embankment-ground surface. Values of the peak displacement at a distance from the track center for different speeds are shown in Figure 13(a). The peak at observation point decrease with the distance from the track center for speeds $60 \mathrm{~m} / \mathrm{s}, 80 \mathrm{~m} / \mathrm{s}, 100 \mathrm{~m} / \mathrm{s}$, and $120 \mathrm{~m} / \mathrm{s}$. For the selected points, the peak displacement increases with the increasing of train speed, but the amplitude is not obvious. Thus, the following conclusion can be obtained. The train speed has no significant effect on peak displacement.

Values of the peak velocity at selected points for different speeds are shown in Figure 13(b). It can be observed that the peak velocity increases with train speed for different points. The peak velocity depends significantly on the distance from the track center, decreases quickly with distance up to $5 \mathrm{~m}$, and slows down from $5 \mathrm{~m}$ to $11 \mathrm{~m}$, then decreases quickly again. The velocity attenuation tendencies in ballast, embankment, and ground are different due to the dynamic character of them.

4.2. Effect of Ballast Modulus. The dynamic response of ballast-embankment-ground is analyzed based on Young's modulus changes of ballast. Time histories of vertical displacement and velocity at point $1.25 \mathrm{~m}$ from the track center for different ballast modulus are shown in Figure 14. 


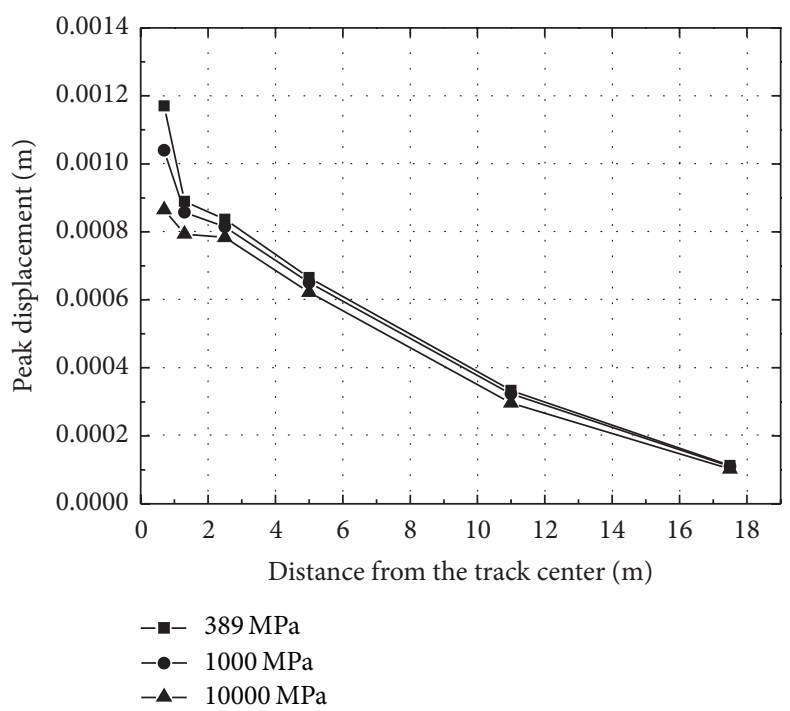

(a) Peak displacement

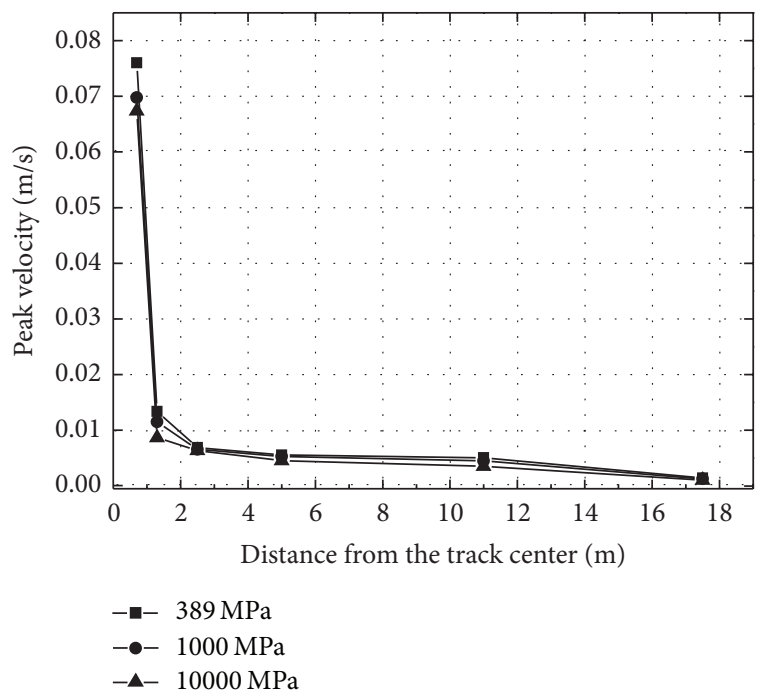

(b) Peak velocity

FIGURE 16: The peak displacement and velocity at a distance from the track center for different ballast modulus.

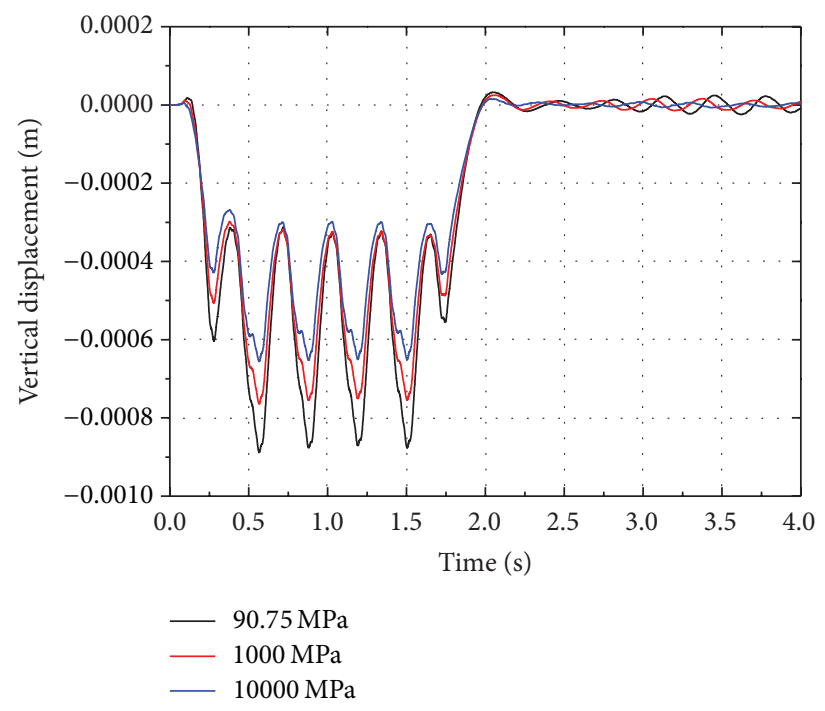

(a) Vertical displacement

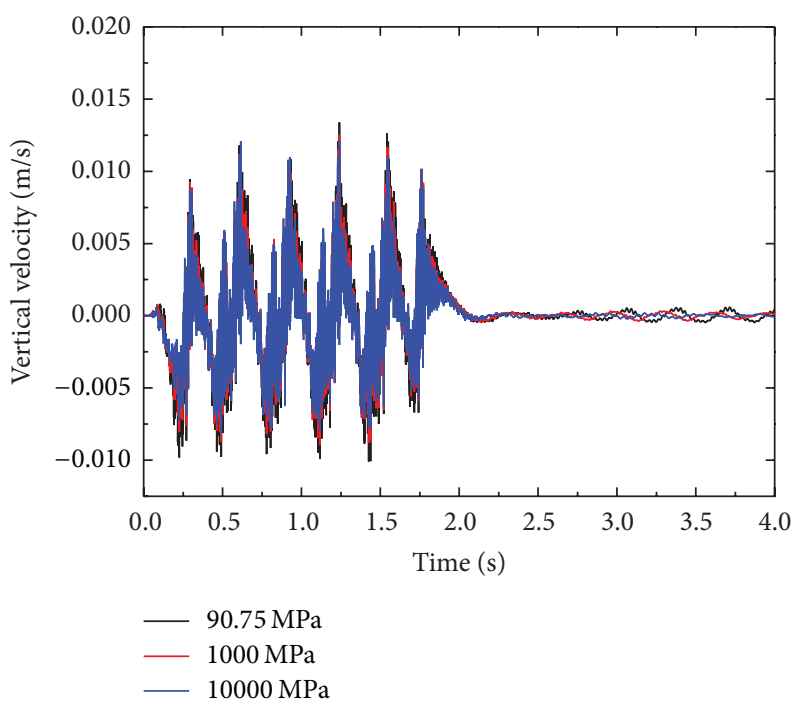

(b) Vertical velocity

FIGURE 17: Time histories of vertical displacement and velocity at point $1.25 \mathrm{~m}$ from the track center for different fill modulus.

The vertical displacement and velocity both decrease with the increase of modulus. Thus, stiffening of ballast can reduce the vibration level and be realized by either installing a concrete slab under the sleeper or replacing the ballast with material with higher stiffness.

The velocity amplitude of Fourier spectrum for different Young's modulus of ballast is shown in Figure 15. It is noted that the velocity amplitude that appears in low, medium, and high frequency ranges decreases with modulus of ballast and tends to move toward high frequency ranges. The peak velocity decreases with the decreasing of modulus, for example, at the value of $10000 \mathrm{MPa}$, moves back to medium frequency ranges, and appears around $80 \mathrm{~Hz}, 140 \mathrm{~Hz}, 170 \mathrm{~Hz}$, and $250 \mathrm{~Hz}$.
The peak displacement and velocity at a distance from the track center are shown in Figure 16. It can be observed from this figure that peak displacement and velocity decrease with the increasing of distance, especially at distances from 1.25 to $2.5 \mathrm{~m}$ away the track center. For example, the peak displacement at location $1.25 \mathrm{~m}$ decreases with the modulus of ballast, but this tendency vanished gradually with the distance away the track center. Similar results can be obtained for the peak velocity with the changing of ballast modulus. For ballast modulus is equal to $389 \mathrm{MPa}$, the peak velocity at the rail is $0.076 \mathrm{~m} / \mathrm{s}$ more larger than that at point on ballast $1.25 \mathrm{~m}$ away the track center, of which the value is $0.013 \mathrm{~m} / \mathrm{s}$. 


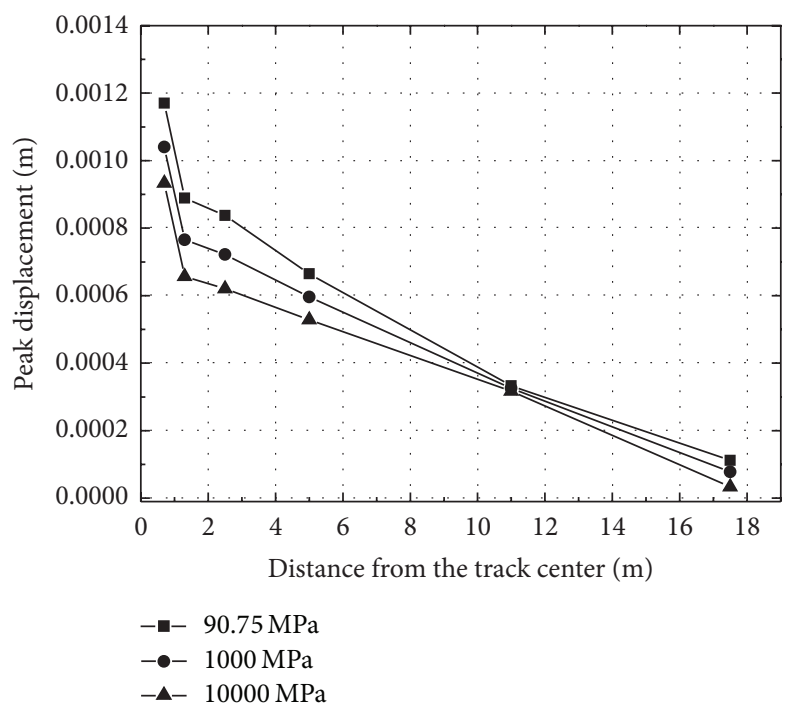

(a) Peak displacement

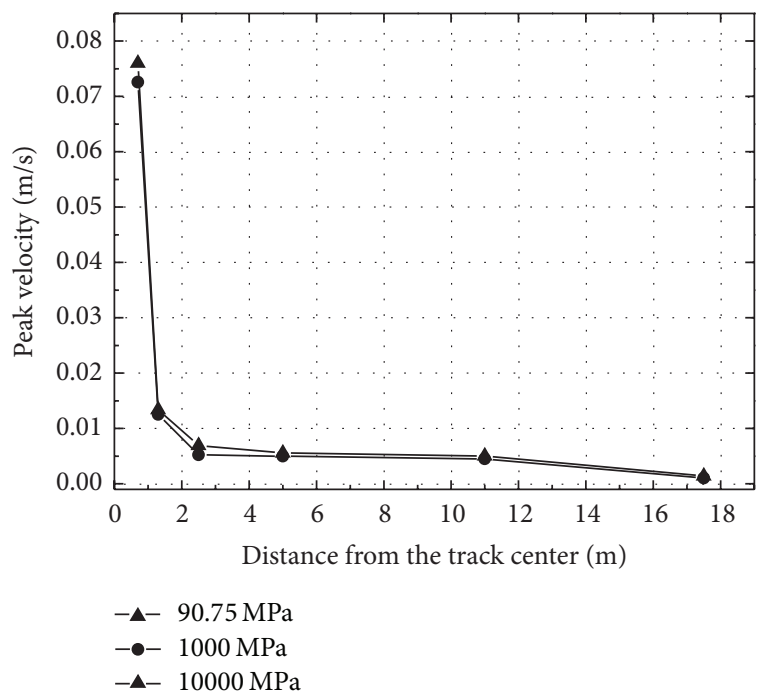

(b) Peak velocity

FIGURE 18: The peak displacement and velocity at a distance from the track center for different fill modulus.

4.3. Effect of Fill Modulus. To instigate the influence of fill, three moduli $90.75,1000$, and $10000 \mathrm{MPa}$ are chosen. The dynamic response of ballast-embankment-ground is analyzed based on Young's modulus variance of fill under the embankment. Time histories of vertical displacement and velocity at point $1.25 \mathrm{~m}$ from the track center are shown in Figure 17. The vertical displacement and velocity both decrease with the increasing of fill modulus. The high value modulus (10000 MPa) may reduce the peak displacement by about $26 \%$ compared to the low value $(90.75 \mathrm{MPa})$. The peak displacement and velocity at a distance from the track center are shown in Figure 18. A reduction of about 26\% for the high-stiffness fill and 15\% for medium-stiffness fill is achieved at point near the track center. But the influence of modulus on peak velocity is not obvious, as seen in Figure 18(b). So stiffening of fill under the embankment can reduce the vibration level, on the other hand, it can be realized by installing a concrete slab under the embankment. Moreover, improvement of soft soil ground is another method to reduce the vibration, which can be achieved by a variety of techniques, such as use of lime-cement piles [47].

Figure 19 shows the effect of improvement of soft soil ground on the vibration produced in the system. In Figure 19(a), the maximum displacement at the rail reduces by about $40 \%$ with the improvement of soft soil, and the maximum displacement at point A $1.25 \mathrm{~m}$ from the track center reduces by about 55\%. In Figure 19(b), the velocity decreases with the improvement of soft soil in accordance. Figure 19(c) shows that the peak velocity decreases with the improvement of soft soil in low frequencies, mainly in the bogie passing frequency $3.2 \mathrm{~Hz}$ and the axle load passage frequency $32 \mathrm{~Hz}$ and tends to move from 32 to $3.2 \mathrm{~Hz}$.

4.4. Effect of Axle Load. Time histories of vertical displacement and velocity at point $1.25 \mathrm{~m}$ from the track center for different axle loads are shown in Figure 20. It can be noted that the vertical displacement and velocity increase with the axle load of high speed train. Compared to axle load $15 t$, the vertical displacement and velocity for axle load $20 t$ increase by about $25 \%$. Thus, the influence of axle load on the vibration of track-embankment-ground system is obviously lower than that of train speed.

Figure 21 shows the peak displacement and velocity at a distance from the track center for different axle loads. The peak displacement decreases with the decreasing of the distance from the track center. The changing law of peak velocity is the same. In Figure 21(b), for the case of axle load $20 t$, the peak velocity at rail is $0.1 \mathrm{~m} / \mathrm{s}$, and the value of which is $0.018 \mathrm{~m} / \mathrm{s}$ at point $1.25 \mathrm{~m}$ from the track center. The influence of axle load on peak displacement and velocity drops gradually with the decreasing of distance. It can be observed that the effective range of vibration from the track center is approximately $2.5 \mathrm{~m}$. The vibration level is low when the dynamic waves reach the embankment.

\section{Conclusions}

In this paper, the finite element analyses of train-induced vibrations presented in this work demonstrate the feasibility of simulating and analyzing the response of trackembankment-ground system. The modeling procedure dealt with the geometric complexities induced by the shape of rail, sleeper, ballast, embankment, and the infinite extension of the natural layered ground. Moreover, the presented numerical model can predict the system vibrations induced by the passing of high speed trains, formulate design recommendations for structures near the tracks, identify the causes of high vibration levels, and evaluate settlements to attenuate them.

The results presented have shown that the numerical model describes the physical phenomenon with excellent 


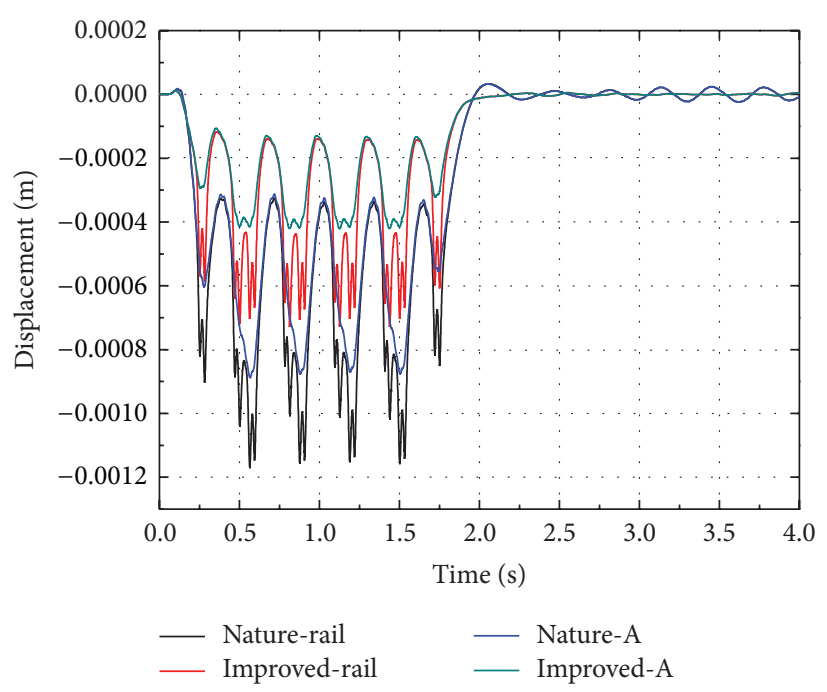

(a) Displacement

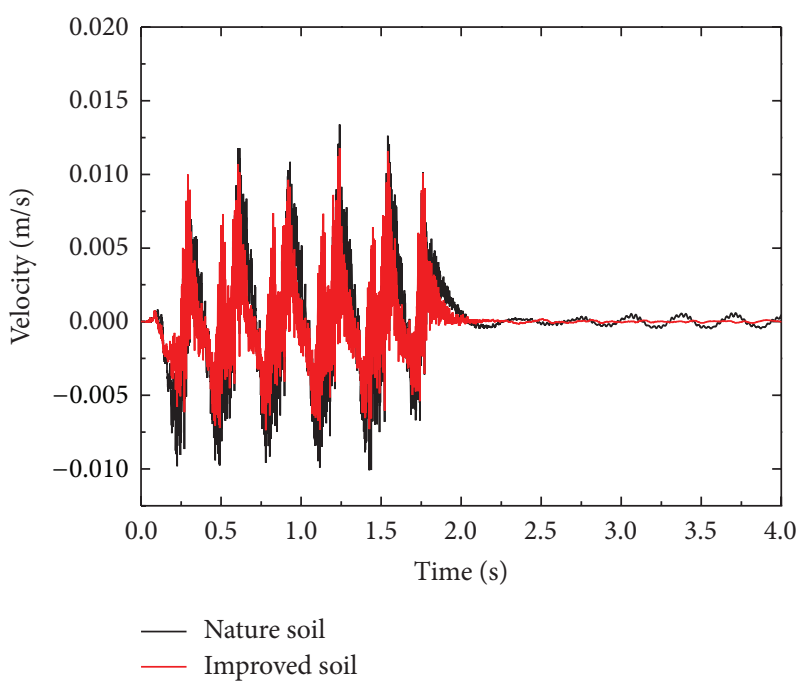

(b) Velocity

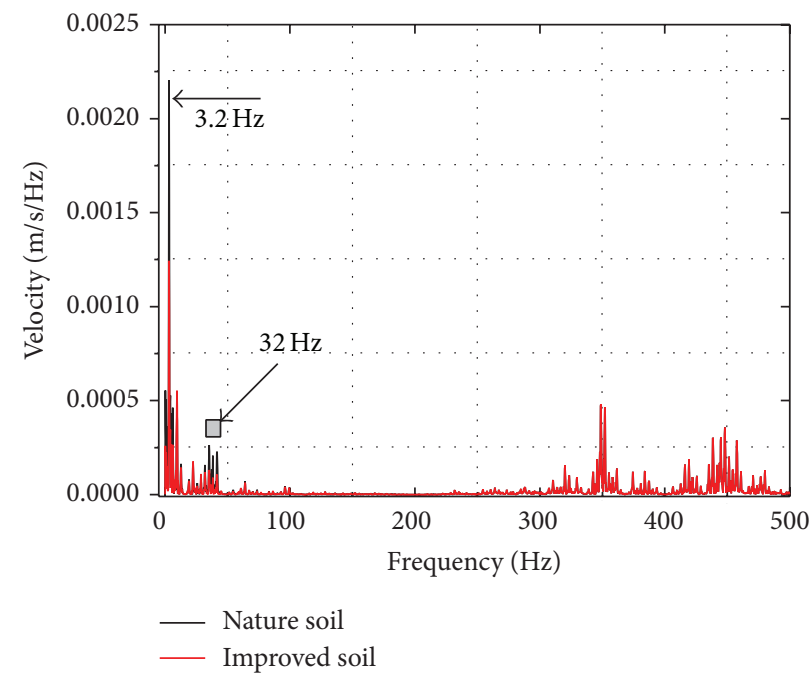

(c) Vertical velocity frequency content

Figure 19: Dynamic response at rail and point A for natural and improved soil.

accuracy. The fundamental components of the frequency content of the response are axle and bogie passing frequency, high-order harmonic frequencies, and the sleeper passing frequency. Also it is noted that the peaks associated with rail and sleeper vibration character in the high frequency range are larger than $200 \mathrm{~Hz}$. The peaks of velocity tend to become high frequency values with the increasing of train speeds. The vibration amplitude of vertical velocity increases with the increasing of the train speed. The velocity attenuation tendencies in ballast, embankment and ground are different due to the dynamic character of them. Stiffening of fill under the embankment can reduce the vibration level, on the other hand, it can be realized by installing a concrete slab under the embankment.

However, in this study, there are no direct validations of those predictions against practical measurements. Only some comparisons are taken out between the numerical results and existing experimental measurements. Further study is needed to investigate the difference between numerical results and measured results to more accurately predict vibrations induced by high speed train loads.

\section{Conflict of Interests}

The authors declare that there is no conflict of interests regarding the publication of this paper.

\section{Acknowledgments}

This work was supported by the National Natural Science Joint High Speed Railway Key Program Foundation of China (Grant no. U1134207) and the 111 Project (Grant no. B13024). 


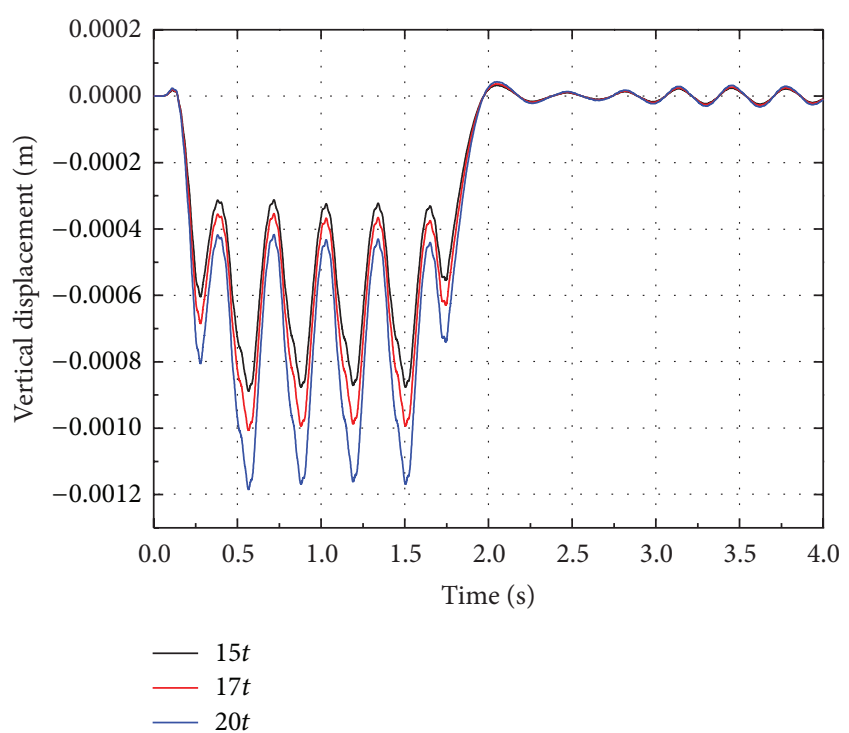

(a)

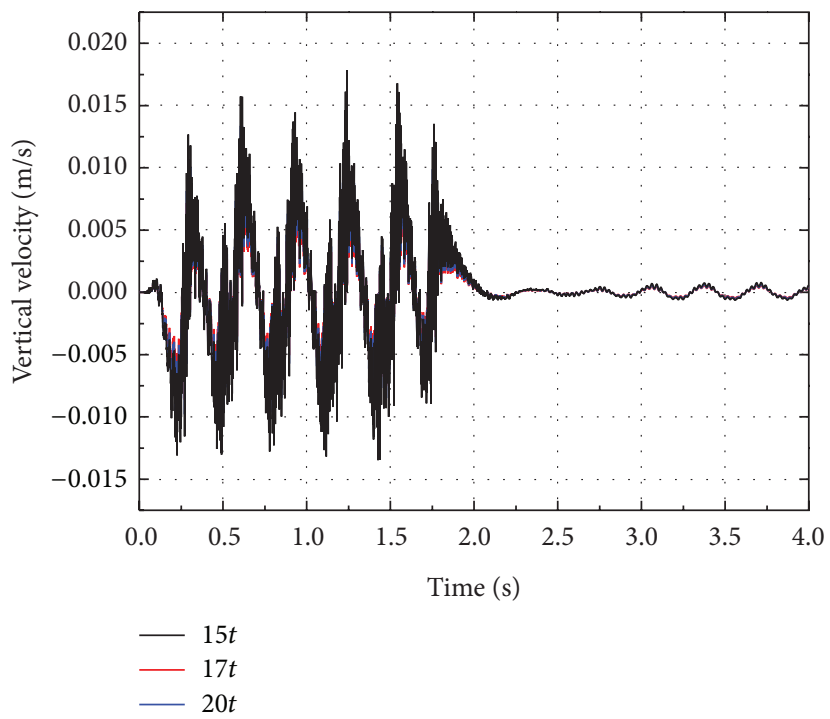

(b)

FIGURE 20: Time histories of vertical displacement and velocity at point $1.25 \mathrm{~m}$ from the track center for different axle loads.

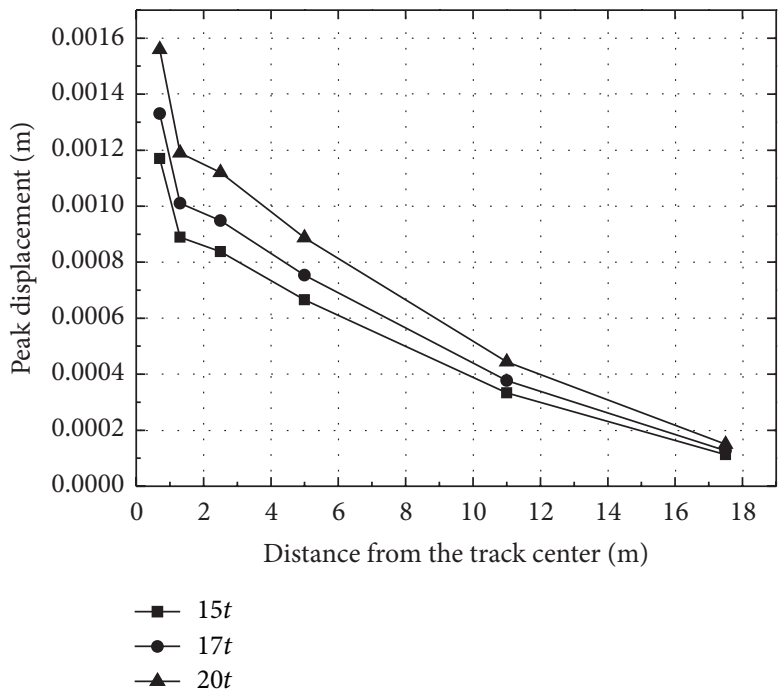

(a)

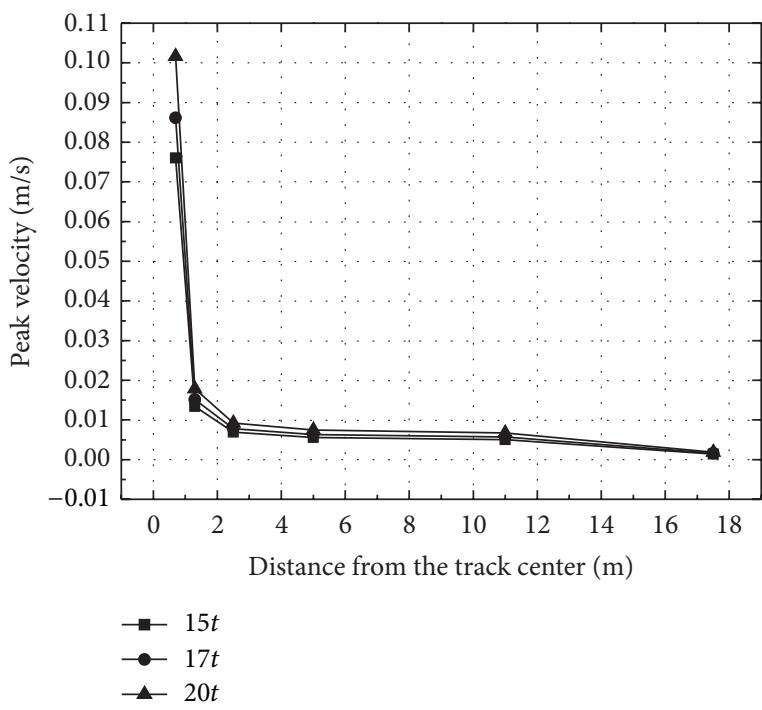

(b)

FIGURE 21: The peak displacement and velocity at a distance from the track center for different axle loads.

\section{References}

[1] C. Madshus and A. M. Kaynia, "High-speed railway lines on soft ground: dynamic behaviour at critical train speed," Journal of Sound and Vibration, vol. 231, no. 3, pp. 689-701, 2000.

[2] A. M. Kaynia, C. Madshus, and P. Zackrisson, "Ground vibration from high-speed trains: prediction and countermeasure," Journal of Geotechnical and Geoenvironmental Engineering, vol. 126 , no. 6, pp. 531-537, 2000.

[3] R. Paolucci, A. Maffeis, L. Scandella, M. Stupazzini, and M. Vanini, "Numerical prediction of low-frequency ground vibrations induced by high-speed trains at Ledsgaard, Sweden," Soil
Dynamics and Earthquake Engineering, vol. 23, no. 6, pp. 425433, 2003.

[4] H. Takemiya, "Simulation of track-ground vibrations due to a high-speed train: the case of X-2000 at Ledsgard," Journal of Sound and Vibration, vol. 261, no. 3, pp. 503-526, 2003.

[5] L. Auersch, "The effect of critically moving loads on the vibrations of soft soils and isolated railway tracks," Journal of Sound and Vibration, vol. 310, no. 3, pp. 587-607, 2008.

[6] B. Xu, J.-F. Lu, and J.-H. Wang, "Dynamic response of an infinite beam overlying a layered poroelastic half-space to moving loads," Journal of Sound and Vibration, vol. 306, no. 1-2, pp. 91110, 2007. 
[7] E. Celebi and G. Schmid, "Investigation of ground vibrations induced by moving loads," Engineering Structures, vol. 27, no. 14, pp. 1981-1998, 2005.

[8] Y. M. Cao, H. Xia, and G. Lombaert, "Solution of moving-loadinduced soil vibrations based on the Betti-Rayleigh Dynamic Reciprocal Theorem," Soil Dynamics and Earthquake Engineering, vol. 30, no. 6, pp. 470-480, 2010.

[9] H. Lamb, "On the propagation of tremors over the surface of an elastic solid," Philosophical Transactions of the Royal Society A, vol. 203, pp. 1-42, 1904.

[10] L. Fryba, Vibrations of Structures Under Moving Loads, Noordhoff International Publishing, Groningen, The Netherlands, 1972.

[11] K. L. Knothe and S. L. Grassie, "Modelling of railway track and vehicle/track interaction at high frequencies," Vehicle System Dynamics, vol. 22, no. 3-4, pp. 209-262, 1993.

[12] V. V. Krylov, "Generation of ground vibrations by superfast trains," Applied Acoustics, vol. 44, no. 2, pp. 149-164, 1995.

[13] G. Degrande, "Free field vibrations during the passage of a high speed train: experimental results and numerical predictions," in Noise and Vibration From High-Speed Trains, V. Krylov, Ed., Thomas Telford Publishing, London, UK, 2001.

[14] P. Galvín and J. Domínguez, "High-speed train-induced ground motion and interaction with structures," Journal of Sound and Vibration, vol. 307, no. 3-5, pp. 755-777, 2007.

[15] A. V. Metrikine and K. Popp, "Vibration of a periodically supported beam on an elastic half-space," European Journal of Mechanics A, vol. 18, no. 4, pp. 679-701, 1999.

[16] X. Sheng, C. J. C. Jones, and D. J. Thompson, "A comparison of a theoretical model for quasi-statically and dynamically induced environmental vibration from trains with measurements," Journal of Sound and Vibration, vol. 267, no. 3, pp. 621-635, 2003.

[17] G. Lombaert, G. Degrande, J. Kogut, and S. François, "The experimental validation of a numerical model for the prediction of railway induced vibrations," Journal of Sound and Vibration, vol. 297, no. 3-5, pp. 512-535, 2006.

[18] L. Auersch, "The effect of critically moving loads on the vibrations of soft soils and isolated railway tracks," Journal of Sound and Vibration, vol. 310, no. 3, pp. 587-607, 2008.

[19] X. Sheng, C. J. C. Jones, and M. Petyt, "Ground vibration generated by a load moving along a railway track," Journal of Sound and Vibration, vol. 228, no. 1, pp. 129-156, 1999.

[20] M. H. Kargarnovin, D. Younesian, D. J. Thompson, and C. J. C. Jones, "Response of beams on nonlinear viscoelastic foundations to harmonic moving loads," Computers and Structures, vol. 83, no. 23-24, pp. 1865-1877, 2005.

[21] L. Sun, "Dynamic displacement response of beam-type structures to moving line loads," International Journal of Solids and Structures, vol. 38, no. 48-49, pp. 8869-8878, 2001.

[22] L. Sun, "A closed-form solution of beam on viscoelastic subgrade subjected to moving loads," Computers and Structures, vol. 80, no. 1, pp. 1-8, 2002.

[23] H. Takemiya and X. Bian, "Substructure simulation of inhomogeneous track and layered ground dynamic interaction under train passage," Journal of Engineering Mechanics, vol. 131, no. 7, pp. 699-711, 2005.

[24] B. Picoux and D. Le Houédec, "Diagnosis and prediction of vibration from railway trains," Soil Dynamics and Earthquake Engineering, vol. 25, no. 12, pp. 905-921, 2005.

[25] H. Sun, Y. Cai, and C. Xu, "Three-dimensional simulation of track on poroelastic half-space vibrations due to a moving point load," Soil Dynamics and Earthquake Engineering, vol. 30, no. 10, pp. 958-967, 2010.

[26] Y. Cai, H. Sun, and C. Xu, "Response of railway track system on poroelastic half-space soil medium subjected to a moving train load," International Journal of Solids and Structures, vol. 45, no. 18-19, pp. 5015-5034, 2008.

[27] Z. G. Cao, Y. Q. Cai, H. L. Sun, and C. J. Xu, "Dynamic responses of a poroelastic half-space from moving trains caused by vertical track irregularities," International Journal for Numerical and Analytical Methods in Geomechanics, vol. 35, no. 7, pp. 761-786, 2011.

[28] Z. Cao and A. Bostromb, "Dynamic response of a poroelastic half-space to accelerating or decelerating trains," Journal of Sound and Vibration, vol. 332, no. 11, pp. 2777-2794, 2013.

[29] G. Y. Gao, Q. S. Chen, J. F. He, and F. Liu, "Investigation of ground vibration due to trains moving on saturated multilayered ground by $2.5 \mathrm{D}$ finite element method," Soil Dynamics and Earthquake Engineering, vol. 40, pp. 87-98, 2012.

[30] X. Sheng, C. J. C. Jones, and D. J. Thompson, "Prediction of ground vibration from trains using the wavenumber finite and boundary element methods," Journal of Sound and Vibration, vol. 293, no. 3-5, pp. 575-586, 2006.

[31] P. Galvín and J. Domínguez, "Analysis of ground motion due to moving surface loads induced by high-speed trains," Engineering Analysis with Boundary Elements, vol. 31, no. 11, pp. 931941, 2007.

[32] P. Galvín and J. Domínguez, "High-speed train-induced ground motion and interaction with structures," Journal of Sound and Vibration, vol. 307, no. 3-5, pp. 755-777, 2007.

[33] P. Galvín, A. Romero, and J. Domínguez, "Fully three-dimensional analysis of high-speed traintracksoil-structure dynamic interaction," Journal of Sound and Vibration, vol. 329, no. 24, pp. 5147-5163, 2010.

[34] L. Hall, "Simulations and analyses of train-induced ground vibrations in finite element models," Soil Dynamics and Earthquake Engineering, vol. 23, no. 5, pp. 403-413, 2003.

[35] S. H. Ju, J. R. Liao, and Y. L. Ye, "Behavior of ground vibrations induced by trains moving on embankments with rail roughness," Soil Dynamics and Earthquake Engineering, vol. 30, no. 11, pp. 1237-1249, 2010.

[36] V. Krylov and C. Ferguson, "Calculation of low-frequency ground vibrations from railway trains," Applied Acoustics, vol. 42, no. 3, pp. 199-213, 1994.

[37] V. V. Krylov, "Generation of ground vibrations by superfast trains," Applied Acoustics, vol. 44, no. 2, pp. 149-164, 1995.

[38] H. Takemiya, "Prediction of ground vibration induced by highspeed train operation," in Proceedings of the 18th Sino-Japan Technology Seminar, pp. 1-10, Chinese Institute of Engineers, 1997.

[39] S. Kaewunruen and A. Remennikov, "Application of vibration measurements and finite element model updating for structural health monitoring of ballasted railtrack sleepers with voids and pockets," in Mechanical Vibration: Measurement, Effect, and Control, R. C. Sapri, Ed., pp. 621-644, Nova Science Publishers, New York, NY, USA, 2009.

[40] S. Kaewunruen and A. M. Remennikov, "Nonlinear transient analysis of a railway concrete sleeper in a track system," International Journal of Structural Stability and Dynamics, vol. 8, no. 3, pp. 505-520, 2008.

[41] J. Lysmer and R. L. Kuhlemeyer, "Finite dynamic model for infinite media," Journal of the Engineering Mechanics Division, vol. 95, no. 4, pp. 859-877, 1969. 
[42] G. Kouroussis, O. Verlinden, and C. Conti, "Finite-dynamic model for infinite media: corrected solution of viscous boundary efficiency," Journal of Engineering Mechanics, vol. 137, no. 7, pp. 509-511, 2011.

[43] T. Belytschko, W. K. Liu, and B. Moran, Nonlinear Finite Elements for Continua and Structures, John Wiley \& Sons, Chichester, UK, 2000.

[44] A. El Kacimi, P. K. Woodward, O. Laghrouche, and G. Medero, "Time domain 3D finite element modelling of train-induced vibration at high speed," Computers and Structures, vol. 118, pp. 66-73, 2013.

[45] X. I. A. He, Traffic Induced Environmental Vibrations and Controls, Science Press, Beijing, China, 2010.

[46] International Standard (ISO) 2631-1, Mechanical Vibration and Shock-Evaluation of Human Exposure to Whole Body Vibration-Part 1: General Requirements, International Organization for Standardization, Geneva, Switzerland, 1997.

[47] K. Karlsrud, "General aspects of transportation infrastructure," in Proceedings of the 12th European Conference on Soil Mechanics and Geotechnical Engineering, vol. 1, pp. 17-30, AA Balkema, Rotterdam, The Netherlands, 1999. 

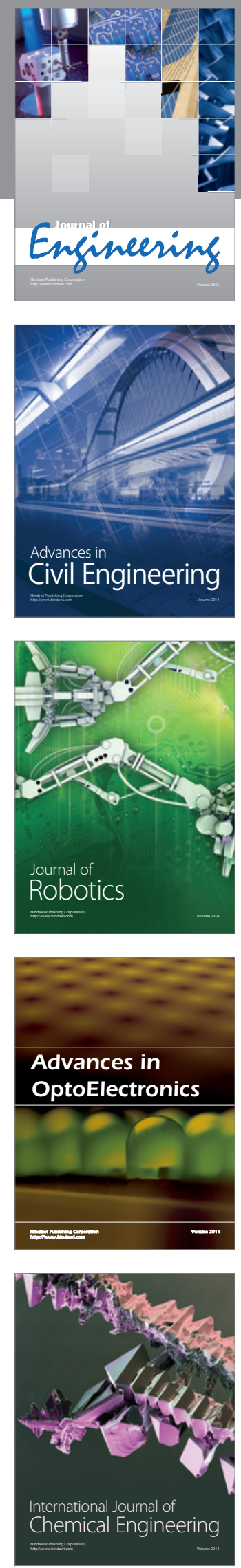

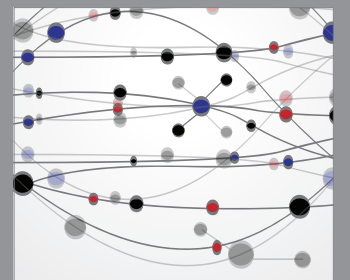

The Scientific World Journal
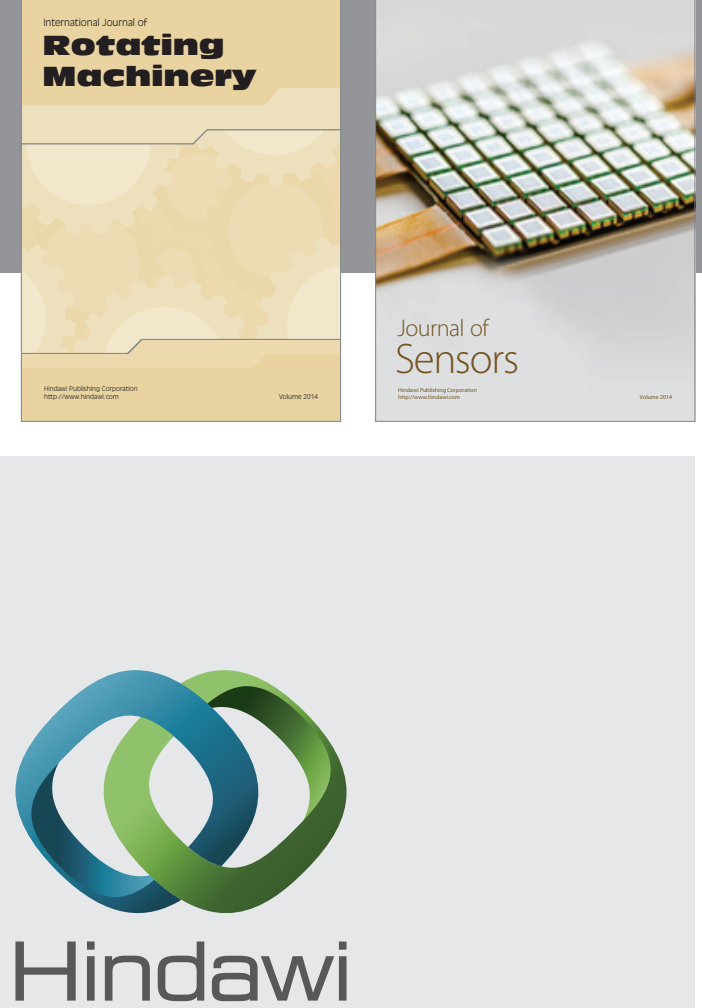

Submit your manuscripts at http://www.hindawi.com
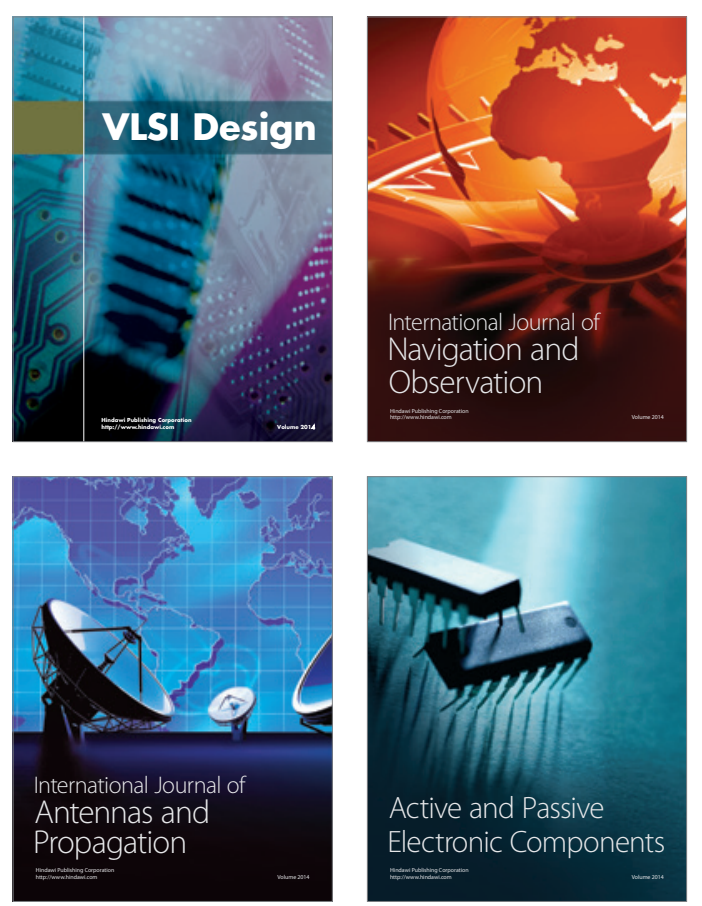
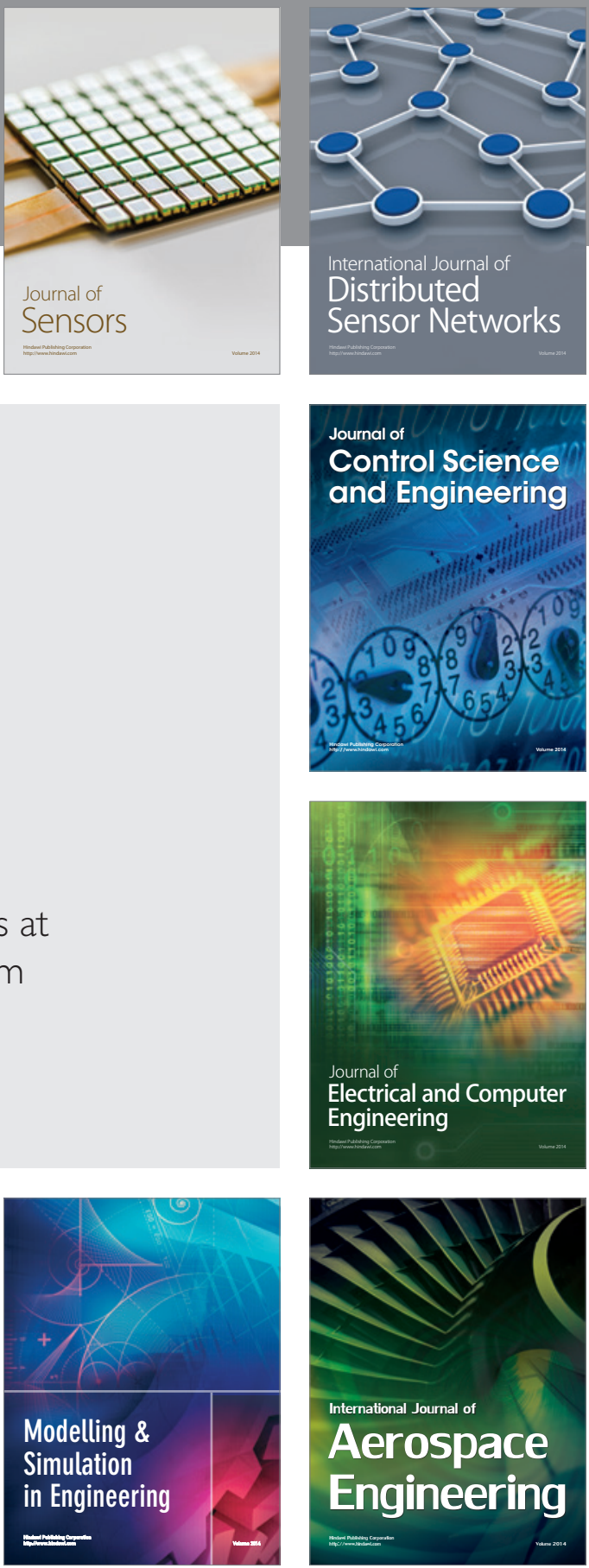

Journal of

Control Science

and Engineering
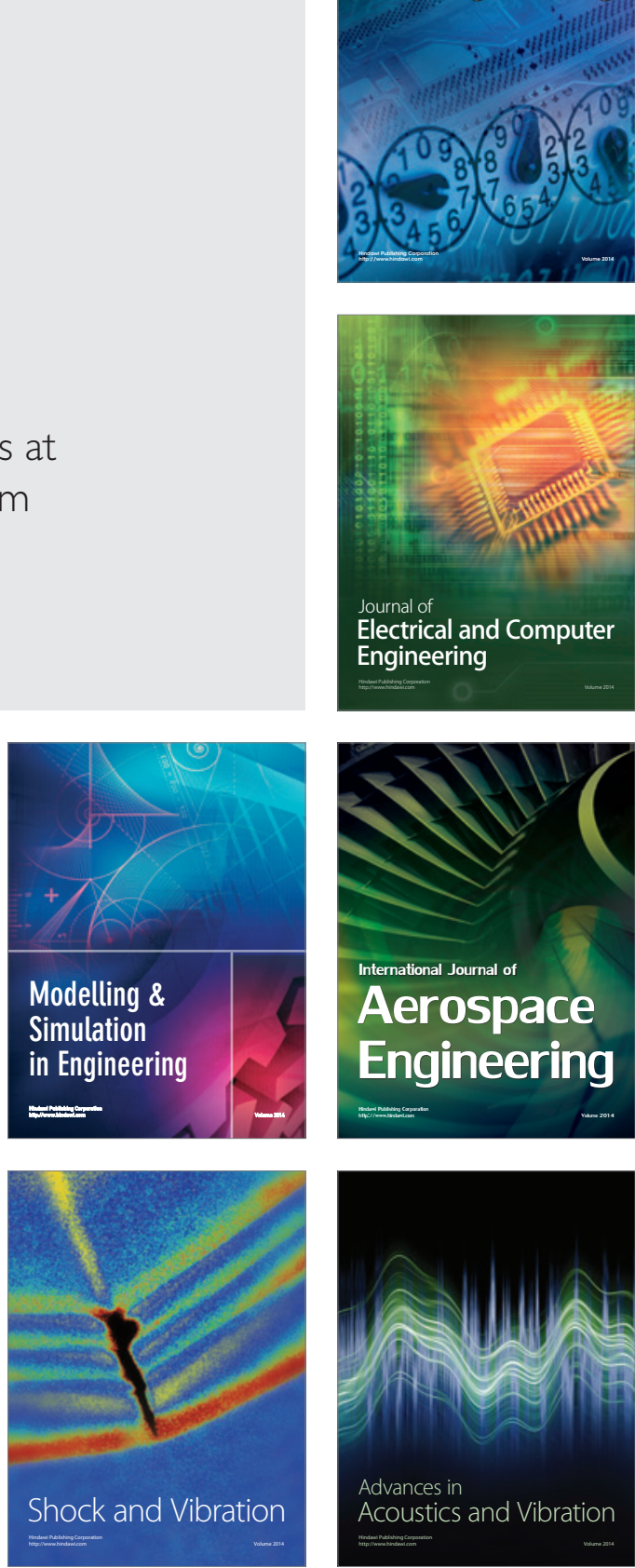